\title{
ASYMPTOTIC BEHAVIOUR OF DENSITIES OF MULTI-DIMENSIONAL STABLE DISTRIBUTIONS
}

By

\author{
Seiji HIRABA
}

\begin{abstract}
In one-dimension asymptotic behaviour of densities of stable distributions is well-known. However, in multi-dimensional cases it is very difficult to investigate asymptotic behaviour of densities of non-degenerate stable distributions in general. In the present paper we give the following two results: If the Lévy measure of the stable distribution has mass at a half-line, then the density decreases along the half-line with the same order as in one-dimensional case. If the Lévy measure is supported only on finitely many halflines, then we can determine asymptotic behaviour along each direction starting at 0 .
\end{abstract}

Keywords: multi-dimensional stable distribution, Lévy-Ito decomposition of Lévy processes.

\section{Introduction and results}

Let $\mu(d x)$ be a stable distribution on $\boldsymbol{R}^{d}$ with exponent $0<\alpha<2$. Then its log-characteristic function $\Psi(z)$ is given as follows: For $z=|z| \xi, \xi \in S^{d-1}=$ $\left\{x \in \boldsymbol{R}^{d}:|x|=1\right\}$,

$$
\begin{aligned}
\Psi(z)= & -|z|^{\alpha} \int_{S^{d-1}}|\langle\xi, \theta\rangle|^{\alpha}\left[1-i \tan \frac{\pi \alpha}{2} \operatorname{sgn}\langle\xi, \theta\rangle\right] \lambda(d \theta)+i\langle z, b\rangle \text { if } \alpha \neq 1, \\
& -|z| \int_{S^{d-1}}|\langle\xi, \theta\rangle|\left[1+i \frac{2}{\pi} \operatorname{sgn}\langle\xi, \theta\rangle \log |\langle z, \theta\rangle|\right] \lambda(d \theta)+i\langle z, b\rangle \text { if } \alpha=1,
\end{aligned}
$$

where $\lambda(d \theta)$ is a finite measure on $\boldsymbol{S}^{d-1}$ and $b \in R^{d}$. If $b=0(\alpha \neq 1)$ or $\int \theta \lambda(d \theta)$ $=0(\alpha=1)$, then $\mu$ satisfies the scaling property: $\mu^{t *}(d x)=t^{-d / \alpha} \mu\left(t^{-1 / \alpha} d x\right)$, in this case $\mu$ is called strictly stable. Note that the Lévy measure $n(d x)$ of $\mu$ is given by

Received January 27, 1993 


$$
n(d x)=\int_{S^{d-1}} \lambda(d \theta) \int_{0}^{\infty} 1_{d x}(r \theta) r^{-1-\alpha} d r \quad \text { on } \quad \boldsymbol{R}^{d} \backslash\{0\} .
$$

We say that $\mu$ is non-degenerate if the support of $\mu$ spans $\boldsymbol{R}^{d}$, or equivalently the support of $\lambda$ spans $\boldsymbol{R}^{d}$. Write this condition Span Spt $\lambda=\boldsymbol{R}^{d}$.

Throughout the present paper we always assume that $\mu$ is a non-degenerate stable distribution on $\boldsymbol{R}^{d}$. It is then well-known that $\mu(d x)$ is absolutely continuous and has a density $p(x)$ with respect to the Lebesgue measure $d x$ on $\boldsymbol{R}^{d}$, which is expressed as

$$
p(x)=\frac{1}{(2 \pi)^{d}} \int_{R^{d}} \exp [-i\langle x, z\rangle+\Psi(z)] d z .
$$

Furthermore $p(x)$ is a $C^{\infty}$-function with derivatives of all orders vanishing at infinity (cf. [6], [7], [8] and [9]).

If we write $p(x)=p(x ; b)$, then $p(x ; b)=p(x-b ; 0)$. Henceforth, we assume $b=0$. Then note that $\mu$ is strictly stable except $\alpha=1$.

We are concerned with asymptotic behaviour of the density function $p(x)$ as $|x| \rightarrow+\infty$. In one-dimension it is well-known that $p(x)$ decreases like $|x|^{-1-\alpha}$ as $x \rightarrow+\infty$ if $\lambda$ has mass at $\{+1\}$. In addition, if $\lambda$ has no mass at $\{-1\}$, then $p(x)=0$ for $x<0$ when $0<\alpha<1$, and $p(x)>0$ for $x<0$ and decreases exponentially fast as $x \rightarrow-\infty$ when $1 \leqq \alpha<2$ (see $\S 2$ ). In multi-dimensional cases Pruitt and Taylor [6] give an upper estimate $p(x) \leqq K|x|^{-1-\alpha}$ for a strictly stable density. When $\lambda$ is absolutely continuous and has a continuous density with respect to the uniform measure on $\boldsymbol{S}^{d-1}$, Dziubanski [2] investigates an asymptotic behaviour $p(r \boldsymbol{\sigma}) \sim c r^{-d-\alpha}$ as $r \rightarrow+\infty$, where $\sigma \in \boldsymbol{S}^{d-1}, \quad c=c(\boldsymbol{\sigma}) \geqq 0$ and $a \sim b$ means that $a / b \rightarrow 1$. Furthermore Arkhipov [1] gives an asymptotic expansion of $p(r \sigma)$ under some additional regularity condition on the density of $\lambda$. On the other hand one can easily deduce that if $\lambda$ is supported only on the orthonormal basis of $\boldsymbol{R}^{d}$, then $p(x)=\Pi_{j=1}^{d} p_{j}\left(x_{j}\right)$, where $x=\left(x_{1}, \cdots, x_{d}\right)$ and $p_{j}$ is a onedimensional density corresponding to $e_{j}$. Therefore if $\sigma \in \boldsymbol{S}^{d-1} \cap\left\{x_{j}>0, j=1\right.$, $\cdots, d\}$, then we have $p(r \sigma) \sim c r^{-d(1+\alpha)}$ as $r \uparrow+\infty$, where $c=c(\sigma)>0$.

From these results it would be expected that a general $\alpha$-stable density $p(r \sigma)$ on $\boldsymbol{R}^{d}$ has the following asymptotic property: For each $\sigma \in \boldsymbol{S}^{d-1}$ there exist $c=c(\sigma)>0$ and $k=k(\alpha, \sigma) \geqq 1+\alpha$ such that

$$
p(r \sigma) \sim c r^{-k} \quad \text { as } r \rightarrow+\infty .
$$

In this paper we first discuss a lower estimate for a general stable density $p(r \sigma)$ and we show that a lower estimate coincides with that of the upper estimate when $\lambda$ has mass at $\sigma$. Furthermore we show that the above asymptotic relation is valid when $\lambda$ is a discrete measure whose support consists of 
only finitely many points in $\boldsymbol{S}^{d-1}$.

Our first result is the following: Let $\mu$ be a non-degenerate stable distribution on $\boldsymbol{R}^{d}$ and Con Spt $\lambda$ be the smallest convex hull in $\boldsymbol{S}^{d-1}$ containing all elements of Spt $\lambda$, and Int $S$ denotes the interior of a set $S$ in $\boldsymbol{S}^{d-1}$. Recall that $b=0$.

THEOREM 1. Suppose that $\lambda$ has mass at $\boldsymbol{\sigma}_{0} \in \boldsymbol{S}^{d-1}$, i.e., $\lambda\left(\left\{\sigma_{0}\right\}\right)>0$. If $0<$ $\alpha<1$ and $\sigma_{0} \in$ Int (Con Spt $\lambda$ ), or if $1 \leqq \alpha<2$, then there exist positive constants $C_{1}=C_{1}\left(\alpha, \sigma_{0}\right)$ and $r_{0}=r_{0}\left(\alpha, \sigma_{0}\right)$ such that $0<C_{1} \leqq r^{1+\alpha} p\left(r \sigma_{0}\right)$ for all $r \geqq r_{0}$, where $C_{1}$ is independent of $r \geqq r_{0}$.

REMARK 1. By the result of [6], assuming thst $\int \theta \lambda(d \theta)=0$ when $\alpha=1$, it holds that $0<C_{1} \leqq r^{1+\alpha} p\left(r \sigma_{0}\right) \leqq C_{2}<\infty$ for all $r \geqq r_{0}$ where the constant $C_{2}$ is independent of $r \geqq 0$ and $\sigma_{0}$ (the upper estimate seems valid without the restriction $\int \theta \lambda(d \theta)=0$ when $\alpha=1$, but we have no proof for it).

Now we assume that $\lambda$ has mass at only finitely many points in $\boldsymbol{S}^{d-1}$ (of course we also assume that $b=0$ and Span Spt $\lambda=\boldsymbol{R}^{d}$ ). To state the next result we define the following subsets of $\boldsymbol{R}^{d}$ : For each $1 \leqq k \leqq d$

(i) $S^{0}(k)$ is a union of closed convex cones with the origin as vertex, the cones which are subtended by every linearly independent $k$-elements of Spt $\lambda$,

(ii) $S(k)=S^{0}(k) \cap S^{d-1}, S(0)=\varnothing$ and $T(k)=S(k)-S(k-1)$.

Now our main result in the present paper is the following:

Theorem 2. Let $d \leqq 3$. Suppose that Spt $\lambda$ is a finite set of $\boldsymbol{S}^{d-1}$. Let $\boldsymbol{\sigma} \in$ $S^{d-1}$.

a) Let $0<\alpha<1$.

If $\sigma \in T(k) \cap$ Int $S(d)$ for some $1 \leqq k \leqq d$, then $p(r \sigma) \sim c_{1} r^{-k(1+\alpha)}$ as $r \rightarrow+\infty$.

If $\boldsymbol{\sigma} \notin$ Int $S(d)$, then $p(r \sigma)=0$.

b) Let $1 \leqq \alpha<2$

If $\sigma \in T(k)$ for some $1 \leqq k \leqq d$, then $p(r \sigma) \sim c_{2} r^{-k(1+\alpha)}$ as $r \rightarrow+\infty$.

If $\sigma \notin S(d)$, then $p(r \sigma)$ decreases faster than any negative order of $r$, that is, $p(r \sigma)$ is a rapidly decreasing function of $r \geqq 0$.

Here constants $c_{1}, c_{2}>0$ are independent of $r$ and can be determined explicitly by the expression of $\Psi(z)$.

For $d \geqq 4$ this theorem could be also proved in a similar way to our proof. However, it seems to be so tedious to describe the proof in general. So we treat the case of $d=2$ and 3. This theorem is proved by using the rotation of 
contour of integration as is similar to the one-dimensional case. Lemmas 2 and 4 are essential to the proof of this theorem (see $\S 3$ ).

In the first cases of (a) and (b) in Theorem 2 we can give more concrete information. We say that $\lambda$ has mass at $(m+1)$-directions $\boldsymbol{\sigma}_{j} \in \boldsymbol{S}^{d-1}, j=0,1,2$, $\cdots, m$, if $\lambda$ has mass at $\sigma_{j}$ and/or $-\sigma_{j}$ for each $j=0,1,2, \cdots, m$ (of course we assume $\sigma_{j} \neq \sigma_{k}$ if $j \neq k$ ). Now suppose that $\lambda$ has mass at only $(m+1)$-directions $\sigma_{j}, j=0,1,2, \cdots, m$. When $\sigma \in T(k)$ for some $1 \leqq k \leqq d$, we define a vertex set $V_{k}(\sigma)$ of $\left\{\sigma_{j}, j=0,1, \cdots, m\right\}$ and an index set $I_{k}(\sigma)$ as follows;

$\left\{\sigma_{j_{1}}, \cdots, \sigma_{j_{k}}\right\} \in V_{k}(\sigma)$ if $\left\{\sigma_{j_{1}}, \cdots, \sigma_{j_{k}}\right\}$ is linearly independent and $\sigma$ is contained in the interior of $\operatorname{Span}\left\{\sigma_{j_{1}}, \cdots, \sigma_{j_{k}}\right\}$,

$$
j(k) \equiv\left\{j_{1}, \cdots, j_{k}\right\} \in I_{k}(\sigma) \quad \text { if } \quad\left\{\sigma_{j_{1}}, \cdots, \sigma_{j_{k}}\right\} \in V_{k}(\sigma) .
$$

Moreover for $j(k) \in I_{k}(\sigma)$ set $H_{j(k)}=\operatorname{Span}\left\{\sigma_{j_{1}}, \cdots, \sigma_{j_{k}}\right\}$ and fix an orthonormal basis $\left\{e_{j_{1}}, \cdots, e_{j_{k}}\right\}$ of $H_{j(k)}$. Now let

(i) $p_{j(k)}$ be a $k$-dimensional density on $H_{j(k)}$ with a log-characteristic function $\left.\Psi\right|_{H_{j(k)}}$,

(ii) $p_{j(k)}^{\perp}$ be a $(d-k)$-dimensional density on $H_{j(k)}^{\frac{1}{j}}$ with a log-characteristic function $\left.\Psi\right|_{H_{j(k)}^{\perp}(i f} k=d$, set $p_{j(k)}^{\perp}=1$ ).

In particular we write $p_{j}=p_{j(1)}$ : a one-dimensional density on $H_{j(1)}$, when $j(1)$ $=\{j\}$.

'THEOREM 3. Let $d \leqq 3$. Suppose that $\sigma \in T(k) \cap \operatorname{Int} S(d)$ in case of $0<\alpha<$ 1 and that $\sigma \in T(k)$ in case of $1 \leqq \alpha<2$ for some $1 \leqq k \leqq d$. Then

$$
\begin{gathered}
p(r \sigma) \sim \sum_{j(k) \in I_{k}(\sigma)} p_{j(k)}(r \sigma(j(k))) p_{j(k)}^{\perp}(0) \quad \text { as } r \rightarrow+\infty, \\
\left.=\sum_{j(k) \in I_{k}(\sigma)} g(j(k))\right) \prod_{s=1}^{k} p_{j_{s}}\left(r h_{j_{s}}\right) p_{j(k)}^{\perp}(0),
\end{gathered}
$$

where $\sigma(j(k))=\sum_{s=1}^{k} h_{j_{s}} \sigma_{j_{s}}=\left.\sigma\right|_{H(k)}$ and $g(j(k))=\left|\operatorname{det} Q_{j(k)}\right|$ with a $k \times k$-matrix $Q_{j(k)}$ such that $Q_{j(k)} \sigma_{j_{s}}=e_{j_{s}}$ for every $s=1,2, \cdots, k$.

Note that the assumption of Theorem 3 implies that there is at least one $j(k)=\left\{j_{1}, \cdots, j_{k}\right\} \in I_{k}(\sigma)$ such that $p_{j(k)}^{1}(0)>0$ and $p_{j_{s}}\left(r \sigma_{j_{s}}\right) \sim c\left(j_{s}\right) r^{-1-\alpha}$ as $r \rightarrow$ $+\infty$ with a positive constant $c\left(j_{s}\right)$ for each $s=1, \cdots, k$.

REMARK 2. a) Note that $S(d)=$ Con Spt $\lambda$ and $T(1)=$ Spt $\lambda$.

b) In a similar way to the proof of Theorem 2 we can show that if $\mu$ is rotation invariant, that is, $\Psi(z)=-c|z|^{\alpha}(c>0)$, then

$$
p(x) \approx \sum_{n=1}^{\infty} c_{n}|x|^{-d-n \alpha} \quad \text { as } \quad|x| \rightarrow+\infty,
$$


where

$$
c_{n}=\pi^{-d / 2-1} \alpha \frac{(-1)^{n-1}}{(n-1) !} 2^{n \alpha-1} c^{n} \sin \frac{\pi n \alpha}{2} \Gamma\left(\frac{n \alpha+d}{2}\right) \Gamma\left(\frac{n \alpha}{2}\right) .
$$

This expansion means that

$$
p(x)=\sum_{n=1}^{N} c_{n}|x|^{-d-n \alpha}+O\left(|x|^{-d-(N+1) \alpha}\right) \quad \text { as } \quad|x| \rightarrow+\infty \text { for all } N .
$$

In particular, if $0<\alpha<1$, then $p(x)=\sum_{n=1}^{\infty} c_{n}|x|^{-d-n \alpha}$.

This result was shown by S.C. Port (A. 13 in [5]) by making use of a subordination technique.

\section{Some Preliminary Results}

For the proof of Theorem 2, we mention some results in the one-dimensional case which are well-known in [3].

a) $\alpha \neq 1$. In this case $p(x)$ is expressed with some constants $c_{0}>0$ and $\left|\beta_{0}\right| \leqq 1$ as follows:

$$
\begin{aligned}
p(x) & =\frac{1}{2 \pi} \int_{-\infty}^{\infty} \exp \left[-i x z-c_{0}|z|^{\alpha}\left(1-i \beta_{0} \tan \frac{\pi \alpha}{2} \operatorname{sgn} z\right)\right] d z \\
& =\frac{1}{2 \pi} \int_{-\infty}^{\infty} \exp \left[-i x z-c|z|^{\alpha} e^{-i \theta} \operatorname{sgn} z\right] d z \\
& \approx \frac{1}{\pi} \sum_{n=1}^{\infty} \frac{(-1)^{n+1}}{n !} x^{-1-n \alpha} \Gamma(n \alpha+1) c^{n} \sin n \eta \text { as } 0<x \rightarrow+\infty,
\end{aligned}
$$

where

$$
\begin{aligned}
& c=c_{0} \sec \theta, \quad \theta=\theta(\beta)=\pi L(\alpha) \beta / 2 \text { and } \eta=\eta(\theta)=\theta+\pi \alpha / 2 \\
& =\pi(\alpha+L(\alpha) \beta) / 2 \text { with } L(\alpha)=\alpha(0<\alpha<1),=\alpha-2(1<\alpha<2) \\
& \text { and } \beta=2 \pi^{-1} L(\alpha)^{-1} \arctan \left(\beta_{0} \tan \pi \alpha / 2\right) .
\end{aligned}
$$

Note that $|\theta|<\pi / 2, c>0,0 \leqq \eta \leqq \pi,|\beta| \leqq 1$ and

(2.4) $\beta_{0}= \pm 1$ if and only if $\beta= \pm 1$ and then $\lambda$ has mass at only $\{ \pm 1\}$ respectively.

In particular if $\beta_{0}=-1$, then $\eta=0(0<\alpha<1),=\pi(1<\alpha<2)$ and it holds that

$$
\begin{aligned}
& \quad p(x)=0 \quad \text { for } \quad x \geqq 0 \text { if } 0<\alpha<1, \\
& \sim \frac{1}{\sqrt{2 \pi(\alpha-1)}}\left(c_{0} \alpha\right)^{-1 /(2 \alpha-2)} x^{(2-\alpha) /(2 \alpha-2)} \exp \left[-(\alpha-1) \alpha^{-\alpha /(\alpha-1)} c_{0}^{-1 /(\alpha-1)} x^{\alpha /(\alpha-1)}\right] \\
& \text { as } 0<x \rightarrow+\infty \text { if } 1<\alpha<2 . \\
& \text { b) } \alpha=1 .
\end{aligned}
$$




$$
\begin{aligned}
p(x) & =\frac{1}{2 \pi} \int_{-\infty}^{\infty} \exp \left[-i x z-c\left(|z|+i \frac{2 \beta}{\pi} z \log |z|\right)\right] d z, c>0,|\beta| \leqq 1, \\
& \approx \frac{1}{\pi} \sum_{n=1}^{\infty} \frac{c^{n}}{n !} x^{-1-n} \int_{0}^{\infty} e^{-z} z^{n} \operatorname{Im}\left[i(1+\beta)-\frac{2 \beta}{\pi} \log \frac{z}{x}\right]^{n} d z \text { as } 0<x \rightarrow+\infty .
\end{aligned}
$$

In this case (2.4) also holds. Moreover, if $\beta=-1$ (i.e., Spt $\lambda=\{-1\}$ ), then

$$
p(x) \sim \frac{1}{2 \sqrt{c e}} \exp \left[\frac{\pi}{4 c} x-\frac{2}{\pi e} c e^{-\pi /(2 c)}\right] \text { as } 0<x \rightarrow+\infty .
$$

c) The asymptotic behaviour of each derivative of $p(x)$ is obtained by differentiating the above formulae.

d) Moreover (cf. [9])

(2.8) $p(x)=0$ if and only if $0<\alpha<1$ and either $x \geqq 0, \beta=-1$ or $x \leqq 0, \beta=1$. In particular if $\alpha \neq 1$ then

$$
p(0)=\pi^{-1} c^{-1 / \alpha} \Gamma\left(\alpha^{-1}+1\right) \cos \left(\frac{\pi}{2 \alpha} L(\alpha) \beta\right) .
$$

REMARK 3. In the case $0>x \rightarrow-\infty$, we obtain the same results by changing $x, \beta_{0}$ and $\beta$ to $|x|,-\beta_{0}$ and $-\beta$ (thus, $\theta$ to $-\theta$ ) respectively. Because if we write $p(x ; \alpha, \beta)=p(x)$ as $p(x)$ depends on $(\alpha, \beta)$, then $p(-x ; \alpha, \beta)=$ $p(x ; \alpha,-\beta)$ holds.

\section{Proof of Results}

Before proceeding to the proof of Theorem 1, we present a general fact on multidimensional stable distributions, which is interesting in its own right. Let $p(x)$ be a density function of non-degenerate stable distribution $\mu$ of exponent $0<\alpha<2$. Recall that $b=0$ in $\Psi(z)$ and $S^{0}(d)$ is the smallest closed convex cone with vertex 0 , which contains Spt $\lambda$. Note that Int $S^{0}(d) \neq \varnothing$ because of Span Spt $\lambda=\boldsymbol{R}^{d}$, where Int $V$ denotes interior of a set $V$ in $\boldsymbol{R}^{d}$.

LEMMA 1. $p(x)=0$ if and only if $0<\alpha<1$ and $x \notin \operatorname{Int} S^{0}(d)$.

Proof. Let $\left(X_{t}, P\right)$ be a Lévy process on $\boldsymbol{R}^{d}$ corresponding to $\mu$, then $P\left(X_{t} \in d x\right)=\mu^{t *}(d x)$. Of course for each $t>0, \mu^{t *}(d x)$ has a $C^{\infty}$-density $p_{t}(x)$ with respect to the Lebesgue measure on $\boldsymbol{R}^{d}$, and $p_{1}=p$. We divide the proof into three cases: $\alpha=1,1<\alpha<2$ and $0<\alpha<1$, and use the Lévy-Ito decomposition of Lévy processes (see [4], [8]).

(1) $\quad \alpha=1$. In this case $\Psi(z)$ is expressed by 


$$
\begin{aligned}
\Psi(z) & =-|z| \int_{S^{d-1}}|\langle\xi, \theta\rangle|\left[1+i \frac{2}{\pi} \operatorname{sgn}\langle\xi, \theta\rangle \log |\langle z, \theta\rangle|\right] \lambda(d \theta) \\
& =\int_{S^{d-1}} \lambda_{0}(d \theta) \int_{0}^{\infty}\left[e^{i\langle z, r \theta\rangle}-1-i\langle z, r \theta\rangle 1_{(0,1)}(r)\right] r^{-2} d r+\left\langle b_{0}, z\right\rangle,
\end{aligned}
$$

where $\lambda_{0}=2 \pi^{-1} \lambda$ and $b_{0}=-2 \pi^{-1} c_{0} \int \theta \lambda(d \theta)$ with

$$
c_{0}=\int_{1}^{\infty} r^{-2} \sin r d r+\int_{0}^{1} r^{-2}(\sin r-r) d r
$$

Then by the Lévy-Ito decomposition we see that

$$
X_{t}=\int_{0}^{t} \int_{0<|x|<1} x \tilde{N}(d s d x)+\int_{0}^{t} \int_{1 \leq|x|<\infty} x N(d s d x)+t b_{0},
$$

where $N(d s d x)=\#\left\{s \in d s: X_{S}-X_{S_{-}} \in d x\right\}$ is a Poisson random measure corresponding to a Poisson point process with characteristic measure

$$
n(d x)=\int_{S^{d-1}} \lambda_{0}(d \theta) \int_{0}^{\infty} 1_{d x}(r \theta) r^{-2} d r \quad \text { on } \quad \boldsymbol{R}^{d} \backslash\{0\}
$$

and $\tilde{N}(d s d x)=N(d s d x)-d s n(d x)$. Now for each $0<\varepsilon<1$ we define

$$
\begin{aligned}
X_{t}^{\varepsilon} & =\int_{0}^{t} \int_{\varepsilon \leqq|x|<1} x \tilde{N}(d s d x)+\int_{0}^{t} \int_{1 \leqq|x|<\infty} x N(d s d x)+t b_{0} \\
& =\int_{0}^{t} \int_{\varepsilon \leqq|x|<\infty} x N(d s d x)-t b^{\varepsilon}
\end{aligned}
$$

with

$$
b^{\varepsilon}=\left(-\log \varepsilon+c_{0}\right) \frac{2}{\pi} \int_{S^{d-1}} \theta \lambda(d \theta)
$$

Then $X_{t}^{\varepsilon}+t b^{\varepsilon}$ is a compound Poisson Process with Lévy measure

$$
n^{\varepsilon}(d x)=\int_{S^{d-1}} \lambda_{0}(d \theta) \int_{\varepsilon}^{\infty} 1_{d x}(r \theta) r^{-2} d r
$$

Thus, if we set $F_{0}^{\varepsilon}=\{0\}, F_{1}^{\varepsilon}=\operatorname{Spt} n^{\varepsilon}, F_{n+1}^{\varepsilon}=F_{n}^{\varepsilon}+F_{1}^{\varepsilon}(n \geqq 1)$, then it holds that Spt $X_{t}^{\varepsilon}+t b^{\varepsilon}=\mathbf{C L}\left(\cup_{n=0}^{\infty} F_{n}^{\varepsilon}\right)$ for all $t>0$ and that $\uparrow \lim _{\varepsilon \downarrow 0} \mathbf{C L}\left(\cup_{n=0}^{\infty} F_{n}^{\varepsilon}\right)=S^{0}(d)$, where Spt $X_{t}^{\varepsilon}$ denotes a support of a distribution of $X_{t}^{\varepsilon}$ under $P$ and CL $V$ denotes closure of a set $V$ in $\boldsymbol{R}^{d}$. From these results we can easily see that Spt $\mu$ $=\boldsymbol{R}^{d}$. In fact, if $\int \theta \lambda(d \theta)=0$ then $S^{0}(d)=\boldsymbol{R}^{d}$ because of Span Spt $\lambda=\boldsymbol{R}^{d}$. Hence Spt $X_{t}=\uparrow \lim _{\varepsilon \downarrow 0}$ Spt $X_{t}^{\varepsilon}=S^{0}(d)=R^{d}$ for all $t>0$. Therefore Spt $\mu=\operatorname{Spt} X_{1}=R^{d}$. If $\int \theta \lambda(d \theta) \neq 0$ then $\left|b^{\varepsilon}\right| \rightarrow+\infty$ as $\varepsilon \rightarrow 0$ and $b^{\varepsilon} \in$ Int $S^{0}(d)$ for small $\varepsilon$ because of $\int \theta \lambda(d \theta) \in$ Int $S^{0}(d)$. Thus for each $x \in \boldsymbol{R}^{d}$ we have $x+b^{\varepsilon} \in \operatorname{Int} S^{0}(d)$ if $0<\varepsilon<1$ is sufficiently small. Hence there is an $0<\varepsilon<1$ such that $x+b^{\varepsilon} \in \mathbf{C L}\left(\cup_{n=0}^{\infty} F_{n}^{\varepsilon}\right)$, that is, $x \in \operatorname{Spt} X_{t}^{\varepsilon} \subset$ Spt $X_{t}$ for all $t>0$. Therefore Spt $\mu=\operatorname{Spt} X_{1}=\boldsymbol{R}^{d}$. Now if 
we assume that $p(x)=0$ for some $x \in \boldsymbol{R}^{d}$, then $L^{*} p(x)=\left.\left(\partial / \partial_{t}\right) p_{t}(x)\right|_{t=1}=0$, where $L^{*}$ is a Lévy generator of $-X_{\iota}$ :

$$
L^{*} p(x)=\int_{S^{d-1}} \lambda_{0}^{*}(d \theta) \int_{0}^{\infty}\left[p(x+r \theta)-p(x)-\langle r \theta, \nabla p(x)\rangle 1_{(0,1)}(r)\right] r^{-2} d r+\left\langle b_{0}, \nabla p(x)\right\rangle
$$

with $\lambda_{0}^{*}(d \theta)=\lambda_{0}(-d \theta)$. Hence noting that $\nabla p(x)=0$, we have $p(x-r \theta)=0$ for a.e. $r \geqq 0$ and $\lambda$-a.e. $\theta \in \mathbf{S p t} \lambda$. By the continuity of $p$ it holds that $p(x-r \theta)=0$ for all $r \geqq 0, \theta \in \operatorname{Spt} \lambda$. Furthermore we easily deduce that

$$
p(x-r \theta)=0 \quad \text { for all } r \geqq 0, \theta \in \operatorname{Con} \text { Spt } \lambda .
$$

This implies that $\mu\left(x\right.$-Int $\left.S^{0}(d)\right)=0$, but which is contrary to Spt $\mu=\boldsymbol{R}^{d}$ and Int $S^{0}(d) \neq \varnothing$. Therefore we get $p(x)>0$ for all $x \in \boldsymbol{R}^{d}$.

(2) $1<\alpha<2$. In this case $p>0$ on $\boldsymbol{R}^{d}$ has been already proved in [9] by using the scaling property of $p_{t}(x)$. We here give an alternative proof by the same way as in (1). In this case the previous arguments work replacing $\Psi(z)$, $n^{\varepsilon}(d x)$ and $L^{*}$ by the following:

$$
\begin{aligned}
\Psi(z) & =-|z|^{\alpha} \int_{S^{d-1}}|\langle\xi, \theta\rangle|^{\alpha}\left[1-i \tan \frac{\pi \alpha}{2} \operatorname{sgn}\langle\xi, \theta\rangle\right] \lambda(d \theta) \\
& =\int_{S^{d-1}} \lambda_{0}(d \theta) \int_{0}^{\infty}\left[e^{i\langle z, r \theta\rangle}-1-i\langle z, r \theta\rangle\right] r^{-1-\alpha} d r,
\end{aligned}
$$

where $\lambda_{0}=c(\alpha) \lambda$ with $c(\alpha)=2 \Gamma(\alpha+1) \sin (\pi \alpha / 2) / \pi$.

$$
X_{t}=\int_{0}^{t} \int_{0<|x|<\infty} x \tilde{N}(d s d x)
$$

with Lévy measure

$$
n(d x)=\int_{S^{d-1}} \lambda_{0}(d \theta) \int_{0}^{\infty} 1_{d x}(r \theta) r^{-1-\alpha} d r \quad \text { on } \quad \boldsymbol{R}^{d} \backslash\{0\} .
$$

For each $0<\varepsilon<1$,

$$
X_{t}^{\varepsilon}=\int_{0}^{t} \int_{s \leq|x|<\infty} x \tilde{N}(d s d x)=\int_{0}^{t} \int_{s \leq|x|<\infty} x N(d s d x)-t b^{\varepsilon}
$$

where

$$
b^{\varepsilon}=\varepsilon^{-\alpha}(\alpha-1)^{-1} \int_{S^{d-1}} \theta \lambda_{0}(d \theta),
$$

and its Lévy measure is given by

$$
n^{c}(d x)=\int_{S^{d-1}} \lambda_{0}(d \theta) \int_{0}^{\infty} 1_{d x}(r \theta) r^{-1-\alpha} d r
$$

The Lévy generator $L^{*}$ of $-X_{t}$ :

$$
L^{*} p(x)=\int_{S^{d-1}} \lambda_{0}^{*}(d \theta) \int_{0}^{\infty}[p(x+r \theta)-p(x)-\langle r \theta, \nabla p(x)\rangle] r^{-1-\alpha} d r .
$$


(3) $0<\alpha<1$. We show that $p(x)=0$ if and only if $x \notin \operatorname{Int} S^{0}(d)$. In this case $\Psi(z)$ and $X_{t}$ are expressed by the following:

$$
\begin{aligned}
\Psi(z) & =-|z|^{\alpha} \int_{S^{d-1}}|\langle\xi, \theta\rangle|^{\alpha}\left[1-i \tan \frac{\pi \alpha}{2} \operatorname{sgn}\langle\xi, \theta\rangle\right] \lambda(d \theta) \\
& =\int_{S^{d-1}} \lambda_{0}(d \theta) \int_{0}^{\infty}\left[e^{i\langle\boldsymbol{z}, r \theta\rangle}-1\right] r^{-1-\alpha} d r,
\end{aligned}
$$

where $\lambda_{0}$ is the same as in (2), and

$$
X_{t}=\int_{0}^{t} \int_{0<|x|<\infty} x N(d s d x) .
$$

Moreover for each $0<\varepsilon<1$ we define

$$
X_{t}^{\varepsilon}=\int_{0}^{t} \int_{\varepsilon \leq|x|<\infty} x N(d s d x),
$$

then Spt $X_{t}^{\varepsilon}=\mathbf{C L}\left(\cup_{n=0}^{\infty} F_{n}^{\varepsilon}\right)$. Hence by limiting $\varepsilon \rightarrow 0$ we have Spt $X_{t}=S^{0}(d)$, that is, $p(x)=0$ if $x \notin \mathbf{I n t} S^{0}(d)$. Furthermore by a similar argument to (1) we can see that $p(x)>0$ if $x \in \operatorname{Int} S^{0}(d)$. In fact, if $p(x)=0$ for some $x \in \operatorname{Int} S^{0}(d)$, then $L^{*} p(x)=\left.(\partial / \partial t) p_{t}(x)\right|_{t=1}=0$, where $L^{*}$ is given by

$$
L^{*} p(x)=\int_{S^{d-1}} \lambda_{0}^{*}(d \theta) \int_{0}^{\infty}[p(x+r \theta)-p(x)] r^{-1-\alpha} d r
$$

with $\lambda_{0}^{*}(d \theta)=\lambda_{0}(-d \theta)$. Hence we have $\mu\left(x-\right.$ Int $\left.S^{0}(d)\right)=0$, but this is contrary to Spt $\mu=S^{0}(d)$. Therefore we get $p>0$ on Int $S^{0}(d)$.

Q.E.D.

We also mention the following result: To emphasize the dependence on $\lambda$ we write $\Psi(z)=\Psi_{\lambda}(z)$ and $p(x)=p_{\lambda}(x)$. Let $Q$ be a linear transformation on $\boldsymbol{R}^{d}$ and set $\lambda_{Q}(d \theta)=\lambda\left(Q^{-1} d \theta\right)$ on $Q\left(\boldsymbol{S}^{d-1}\right)$. Then by the definition of $\Psi(z)$ we have $\Psi_{\lambda_{Q}}(z)=\Psi_{\lambda}\left({ }^{t} Q z\right)$, where ${ }^{t} Q$ denotes a transposed matrix of $Q$. Moreover by using (1.1) we can easily deduce that if $Q$ is invertible, then $p_{\lambda_{Q}}$ is welldefined and

$$
p_{\lambda}(x)=|\operatorname{det} Q| p_{\lambda_{Q}}(Q x)
$$

holds.

Proof of THEOREM 1. First assume that $\lambda\left(\left\{\sigma_{0}\right\}\right)>0$ for some $\sigma_{0} \in \boldsymbol{S}^{d-1}$, and also that $\sigma_{0} \in \operatorname{Int}\left(\right.$ Con Spt $\lambda$ ) if $0<\alpha<1$. For simplicity we write $\sigma_{0}=\sigma$. In (3.1) let $Q$ be an orthogonal transformation, then $p_{\lambda}(x)=p_{\lambda_{Q}}(Q x)$. From this we may assume that $\sigma=(1,0, \cdots, 0)$. Moreover it is easily deduced that $p(r \sigma)$ is expressed by

$$
p(r \sigma)=c p_{1}(r) p_{d-1}(0, \cdots, 0)
$$


or

$$
p(r \sigma)=\int_{-\infty}^{\infty} p_{1}(r-y) p_{d}(y, 0, \cdots, 0) d y,
$$

where $p_{j}$ is a $j$-dimensional density $(j=1, d-1, d)$ and $c>0$. In fact, we define $\lambda^{\sigma}$ by $\lambda=\delta_{(\sigma)}+\lambda^{\sigma}$ and set $H=$ Span Spt $\lambda^{\sigma}$. Then $\operatorname{dim} H=d-1$ or $d$ because of Span Spt $\lambda=\boldsymbol{R}^{d}$. If $\operatorname{dim} H=d-1$, then by taking $Q$ in (3.1) such that $Q \boldsymbol{\sigma}=\boldsymbol{\sigma}$ and $Q(H)=\left\{x_{1}=0\right\}$ we see that $p_{\lambda_{Q}}(r \sigma)=p_{1}(r) p_{d-1}(0, \cdots, 0)$, where $p_{1}$ (resp. $\left.p_{d-1}\right)$ is a one-dimensional density function (resp. $(d-1)$-dimensional density function) corresponing to $\delta_{|\sigma|}\left(\right.$ resp. $\left.\lambda_{Q}^{\sigma}\right)$. Hence we get $p(r \sigma)=|\operatorname{det} Q| p_{1}(r) p_{d-1}(0, \cdots, 0)$. If $\operatorname{dim} H=d$, then we can define a $d$-dimensional density function $p_{d}$ by $\lambda^{\sigma}$. Thus we have

$$
\begin{aligned}
(2 \pi)^{d} p(x) & =\int_{R^{d}} \exp \left[-i\langle x, z\rangle+\Psi_{\delta_{|\sigma|}}(z)+\Psi_{\lambda^{\sigma}}(z)\right] d z \\
= & \int_{-\infty}^{\infty} d y p_{1}(y) \int_{R^{d}} \exp \left[-i\left\{\left(x_{1}-y\right) z_{1}+x_{2} z_{2}+\cdots+x_{d} z_{d}\right\}+\Psi_{\lambda^{\sigma}}(z)\right] d z \\
& =(2 \pi)^{d} \int_{-\infty}^{\infty} p_{1}(y) p_{d}\left(x_{1}-y, x_{2}, \cdots, x_{d}\right) d y .
\end{aligned}
$$

Therefore (3.3) holds. Here in the second equation we use

$$
\exp \left[\Psi_{\delta_{|\sigma|}}(z)\right]=\int_{-\infty}^{\infty} p_{1}(y) \exp \left[i y z_{1}\right] d y .
$$

Now noting that (3.2) does not occur when $0<\alpha<1$ and Con Spt $\lambda \neq \boldsymbol{S}^{d-1}$, we see that $p_{d-1}(0, \cdots, 0)>0$ and $p_{d}(y, 0, \cdots, 0)>0$ if at least $y>0$ by Lemma 1. Hence in the case of (3.2) our claim holds. In the case of (3.3) we have $p(r \sigma) \geqq c p_{1}(2 r)$ for sufficiently large $r$ with a positive constants $c$. In fact there are a compact set $K$ in $(0, \infty)$ and a positive constant $r_{0}$ such that $\varepsilon \equiv$ $\inf _{y \in K} p_{d}(y, 0, \cdots, 0)>0$ and $\inf _{y \in K} p_{1}(r-y) \geqq p_{1}(2 r)$ for all $r \geqq r_{0}$. Thus $p(r \sigma) \geqq$ $\varepsilon|K| \inf _{y \in K} p_{1}(r-y) \geqq \varepsilon|K| p_{1}(2 r)$ for $r \geqq r_{0}$. Since $p_{1}(2 r) \sim c^{\prime} r^{-1-\alpha}$ as $r \rightarrow+\infty$, there is a constant $C_{1}>0$ such that $p(r \sigma) \geqq C_{1} r^{-1-\alpha}$ for all $r \geqq r_{0}$. $\quad$ Q.E.D.

Proof of Theorem 2 AND Theorem 3. Let $d=2,3$ and let $\mu$ be a nondegenerate stable distribution on $\boldsymbol{R}^{d}$ with exponent $0<\alpha<2$. Recall that we are assuming that Spt $\lambda$ is a finite set of $\boldsymbol{S}^{d-1}$, and we say that $\lambda$ has mass at $(m+1)$-directions $\sigma_{j} \in S^{d-1}, j=0,1,2, \cdots, m$, if $\lambda$ has mass at $\sigma_{j}$ and/or $-\sigma_{j}$ for each $j=0,1,2, \cdots, m$ (of course $\sigma_{j} \neq \pm \sigma_{k}$ if $j \neq k$ ).

Now we begin with the case $d=2$. The proof is divided into three cases.

CASE 1. $\lambda$ has mass at only two directions $\sigma_{0}, \sigma_{1}\left(\sigma_{0} \neq \pm \sigma_{1}\right)$. By (3.1) we 
may assume that $\sigma_{0}=(1,0), \sigma_{1}=(a, b)$ and with $a \neq 1, b>0$ such that $a^{2}+b^{2}=1$. Then

$$
p(r \sigma)=b^{-1} p_{0}\left(r h_{0}\right) p_{1}\left(r h_{1}\right)
$$

where, $h_{j}$ are defined by the decomposition $\sigma=h_{0} \sigma_{0}+h_{1} \sigma_{1}$, and $p_{j}(y), y \in \boldsymbol{R}$ are defined by (2.1) with some constants $\left(c_{j, 0}, \beta_{j, 0}\right)$ instead of $\left(c_{0}, \beta_{0}\right), j=0,1$. Here one can easily check that $b^{-1}=g(\{0,1\})$; which is defined in Theorem 3 , and that $p_{0}^{\perp}(0)=b^{-1} p_{1}(0)$ and $p_{1}^{\perp}(0)=b^{-1} p_{0}(0)$. Hence our claim immediately follows by using the facts (2.2), (2.4), (2.8) and (2.9). In particular if $1<\alpha<2$ and $\sigma \notin$ Con Spt $\lambda$, then by (2.5) and (2.7),

$$
\begin{aligned}
& p(r \sigma) \sim K_{1} r^{K_{2}} \exp \left[-K_{3} r^{K_{4}}\right] \quad \text { as } \quad r \rightarrow+\infty \text { if } 1<\alpha<2, \\
& p(r \sigma) \sim \widetilde{K}_{1} \exp \left[\tilde{K}_{2} r-\widetilde{K}_{3} e^{\tilde{K}_{4} r}\right] \quad \text { as } r \rightarrow+\infty \text { if } \alpha=1,
\end{aligned}
$$

where $K_{j}, \tilde{K}_{j}$ are positive constants which are independent of $r$. For instance, when Spt $\lambda=\left\{ \pm \sigma_{0}, \sigma_{1}\right\}$ with $\sigma_{0}=(1,0)$ and $\sigma_{1}=(0,1)$, let $\sigma=(s, t)$,

if $\sigma \in T(2)$, i.e., $t>0$ and $\sigma \neq \sigma_{1}$, then $p(r \sigma) \sim c r^{-2(1+\alpha)}$ as $r \rightarrow+\infty$;

if $\sigma \in T(1) \cap$ Int $S(2)$, i.e., $\sigma=\sigma_{1}$, then $p(r \sigma) \sim c r^{-(1+\alpha)}$ as $r \rightarrow+\infty$;

if $\sigma \in T(1) \cap \partial S(2)$, i.e., $\sigma= \pm \sigma_{0}$, then $p(r \sigma)=0(0<\alpha<1), p(r \sigma) \sim c r^{-(1+\alpha)}(1 \leqq$ $\alpha<2)$ as $r \rightarrow+\infty$;

if $\sigma \notin S(2)$, i.e., $t<0$, then $p(r \sigma)=0$ for all $r \geqq 0(0<\alpha<1)(3.4)(1<\alpha<2)$ and (3.5) $(\alpha=1)$ hold.

CASE 2. $\alpha \neq 1$ and $\lambda$ has mass at only $(m+1)$-directions $\sigma_{j}, j=0,1,2, \cdots$, $m(m \geqq 2)$. Then $\Psi(z), z=\left(z_{1}, z_{2}\right)$, is expressed by

$$
\begin{aligned}
\Psi(z) & =-\sum_{j=0}^{m} c_{j, 0}\left|\left\langle\sigma_{j}, z\right\rangle\right|^{\alpha}\left[1-i \beta_{j, 0} \tan \frac{\pi \alpha}{2} \operatorname{sgn}\left\langle\sigma_{j}, z\right\rangle\right] \\
& =-\sum_{j=0}^{m} c_{j}\left|\left\langle\sigma_{j}, z\right\rangle\right|^{\alpha} \exp \left[-i \theta_{j} \operatorname{sgn}\left\langle\sigma_{j}, z\right\rangle\right],
\end{aligned}
$$

where $c_{j, 0}>0,\left|\beta_{j, 0}\right| \leqq 1$ and $c_{j}, \theta_{j}$ are defined by (2.3).

In order to prove Theorem 2 and Theorem 3 in Case 2 we first consider the special case, however we show that the general case is reduced to this special one (see Second step).

First step. Set $\sigma=\sigma_{0}=(1,0)$ and let $\sigma_{j}=\left(s_{j}, t_{j}\right), j=0,1,2, \cdots, m$, where $s_{j}=\cos \varphi_{j}$ and $t_{j}=\sin \varphi_{j}$ with $0=\varphi_{0}<\varphi_{1}<\cdots<\varphi_{m}=\pi / 2$. Note that if $\lambda$ has no mass at $\sigma=(1,0)$, then $\lambda$ has mass at $-\sigma=(-1,0)$ by our definition of directions, and $\beta_{0,0}=-1$.

We define the following $\alpha$-stable densities :

(i) For $y, z \in \boldsymbol{R}, p_{0}(y)$ (resp. $p_{0}^{\perp}(y)$ ) is a one-dimensional density with a 
$\log$-characteristic function $\Psi_{0}(z)=-c_{0}|z|^{\alpha} \exp \left[-i \theta_{0} \operatorname{sgn} z\right]\left(\right.$ resp. $\left.\Psi_{0}^{\perp}(z)=\Psi(0, z)\right)$

(ii) For $x, z \in \boldsymbol{R}^{2}$ and $j \neq k, p_{j, k}(x)$ is a two-dimensional density with a $\log$-characteristic function $\Psi_{j, k}(z)=-\sum_{r=j, k} c_{r}\left|\left\langle\sigma_{r}, z\right\rangle\right|^{\alpha} \exp \left[-i \theta \operatorname{sgn}\left\langle\sigma_{r}, z\right\rangle\right]$.

PROPOSITION. Let $r \geqq 0$.

a) If $\sigma \in$ Spt $\lambda$ and $p_{0}^{\perp}(0)>0$, then

$$
p(r \sigma) \sim p_{0}(r) p_{0}^{\perp}(0) \quad \text { as } \quad r \rightarrow+\infty ;
$$

b) If $\sigma \notin$ Spt $\lambda$ and $\sigma \in$ Con Spt $\lambda$, then

$$
p(r \sigma) \sim \sum_{1 \leq j<k \leq m} p_{j, k}(r \sigma) \quad \text { as } r \rightarrow+\infty ;
$$

c) If $1 \leqq \alpha<2$ and $\sigma \notin$ Con Spt $\lambda$, then $p(r \sigma)$ is rapidly decreasing as $r \rightarrow+\infty$;

d) If $0<\alpha<1$ and $\sigma \notin$ Int (Con Spt $\lambda$ ), then $p(r \sigma)=0$.

Note that (b), (c) and (d) also hold in the case that $\lambda$ has no mass at $\{ \pm \sigma\}$ (in this case $c_{0,0}=c_{0}=0$ in $\left.\Psi(z)\right)$ and that, by $(2.9)$

$$
\operatorname{Re} \int_{0}^{\infty} \exp \Psi\left(0, z_{2}\right) d z_{2}=\pi p_{0}^{\perp}(0)=\bar{c}^{-1 / \alpha} \Gamma\left(\alpha^{-1}+1\right) \cos \left(\frac{\pi}{2 \alpha} L(\alpha) \bar{\beta}\right),
$$

where $(\bar{c}, \bar{\beta})$ is $(c, \beta)$ in $(2.3)$ which is given by using $\left(\bar{c}_{0}, \bar{\beta}_{0}\right)=\left(\sum_{j=1}^{m} c_{j, 0} t_{j}^{\alpha}\right.$, $\left.\sum_{j=1}^{m} c_{j, 0} \beta_{j, 0} t_{j}^{\alpha} / \bar{c}_{0}\right)$ instead of $\left(c_{0}, \beta_{0}\right)$ in $(2.3)$. Hence by (2.4) and (2.8) $p_{0}^{\perp}(0)=0$ if and only if $0<\alpha<1$ and $\beta_{1,0}=\beta_{2,0}=\cdots=\beta_{m, 0}= \pm 1$ (i.e., $\sigma \notin$ Int (Con Spt $\lambda$ )).

From this proposition we can easily deduce Theorem 2 and Theorem 3 in Case 2 by using the one-dimensional results.

To prove Proposition we need some lemmas. The following lemma is obtained by elementary analysis.

LEMMA 2. Set $a_{j}=t_{j} / s_{j}=\tan \phi_{j}\left(a_{0}=0, a_{m}=\infty\right)$. Then

$$
\begin{aligned}
& p(r \sigma)=(2 \pi)^{-2} \int_{R^{2}} \exp \left[-i r z_{1}+\Psi(z)\right] d z \\
& \approx r^{-1} \pi^{-2} \sum_{n=1}^{\infty} \frac{(-1)^{n-1}}{n !} r^{-n \alpha} c_{0}^{n} \sin n \eta_{0} \int_{0}^{\infty} d u e^{-u} u^{n \alpha} \operatorname{Re} \int_{u /\left(r a_{1}\right)}^{\infty} \exp \Psi\left(-i \frac{u}{r}, \nu\right) d \nu \\
& +r^{-2} \pi^{-2} \sum_{n=1}^{\infty} \frac{(-1)^{n-1}}{n !} r^{-n \alpha} c_{0}^{n} \sin n \eta_{0} \int_{0}^{\infty} d u e^{-u} u^{n \alpha+1} \\
& \quad \quad \int_{0}^{\pi / 2} d \phi e^{i \phi} \sum_{n=0}^{\infty} \frac{(-1)^{n}}{n !} r^{-n \alpha} \operatorname{Im}\left[\sum_{j=1}^{m} c_{j} u^{\alpha}\left(s_{j}+i e^{i \phi} t_{j} / a_{1}\right)^{\alpha} e^{-i \eta_{j}}\right]^{n} \\
& \quad+r^{-2} \pi^{-2} \sum_{j=1}^{m-1} \int_{0}^{\infty} d \nu \int_{a_{j} \nu}^{a_{j+1} \nu} d u e^{-u}
\end{aligned}
$$




$$
\begin{aligned}
& \sum_{n=1}^{\infty} \frac{(-1)^{n-1}}{n !} r^{-n \alpha} \operatorname{Im}\left[c_{0} u^{\alpha} e^{-i \eta_{0}}+c_{1} s_{1}^{\alpha}\left(u-a_{1} \nu\right)^{\alpha} e^{-i \eta_{1}}+\cdots\right. \\
& \left.+c_{j} s_{j}^{\alpha}\left(u-a_{j} \nu\right)^{\alpha} e^{-i \eta_{j}}\right]^{n} \\
& \sum_{n=1}^{\infty} \frac{(-1)^{n-1}}{n !} r^{-n \alpha} \operatorname{Im}\left[c_{j+1} s_{j+1}^{\alpha}\left(a_{j+1} \nu-u\right)^{\alpha} e^{-i \hat{\eta}_{j+1}}+\cdots+c_{m} \nu^{\alpha} e^{-i \hat{\eta}_{m}}\right]^{n}
\end{aligned}
$$

as $r \rightarrow+\infty$, where $\eta_{j}=\eta\left(\theta_{j}\right), \hat{\eta}_{j}=\eta\left(-\theta_{j}\right)$ are defined by (2.3). This expansion holds in equal provided $0<\alpha<1$, and if $1<\alpha<2$, then it holds in the sense of (1.2).

Proof. For simplicity we only prove the case that $m=3, \sigma=\sigma_{0}=(1,0)$, $\sigma_{1}=\left(s_{1}, t_{1}\right), \sigma_{2}=\left(s_{2}, t_{2}\right)$ and $\sigma_{3}=(0,1)$. That is, for $\tilde{c}_{j}=c_{j} s_{j}^{\alpha}(j=1,2)$,

$$
\begin{aligned}
\Psi(z)= & -c_{0}\left|z_{1}\right|^{\alpha} \exp \left[-i \theta_{0} \operatorname{sgn} z_{1}\right]-\tilde{c}_{1}\left|z_{1}+a_{1} z_{2}\right|^{\alpha} \exp \left[-i \theta_{1} \operatorname{sgn}\left(z_{1}+a_{1} z_{2}\right)\right] \\
& -\tilde{c}_{2}\left|z_{1}+a_{2} z_{2}\right|^{\alpha} \exp \left[-i \theta_{2} \operatorname{sgn}\left(z_{1}+a_{2} z_{2}\right)\right]-c_{3}\left|z_{2}\right|^{\alpha} \exp \left[-i \theta_{3} \operatorname{sgn} z_{2}\right] .
\end{aligned}
$$

Then

$$
\begin{aligned}
p(r \sigma)= & \frac{\operatorname{Re}}{2 \pi^{2}} \int_{0}^{\infty} d z_{2} \int_{0}^{\infty} d z_{1} \exp \left[-i r z_{1}-c_{0} z_{1}^{\alpha} e^{-i \theta_{0}}\right] \\
& \left(\exp \left[-\tilde{c}_{1}\left(z_{1}+a_{1} z_{2}\right)^{\alpha} e^{-i \theta_{1}}-\tilde{c}_{2}\left(z_{1}+a_{2} z_{2}\right)^{\alpha} e^{-i \theta_{2}}-c_{3} z_{2}^{\alpha} e^{-i \theta_{3}}\right]\right. \\
& +\exp \left[-\tilde{c}_{1}\left|z_{1}-a_{1} z_{2}\right|^{\alpha} e^{-i \theta_{1} \operatorname{sgn}\left(z_{1}-a_{1} z_{2}\right)}\right. \\
& \left.\left.-\tilde{c}_{2}\left|z_{1}-a_{2} z_{2}\right|^{\alpha} e^{-i \theta_{2} \operatorname{sgn}\left(z_{1}-a_{\left.2_{2} z_{2}\right)}\right.}-c_{3} z_{2}^{\alpha} e^{i \theta_{s}}\right]\right) .
\end{aligned}
$$

By changing variable $r z_{1}$ to $u$ we have

$$
\begin{aligned}
& 2 \pi^{2} r p(r \sigma)=\operatorname{Re} \int_{0}^{\infty} d z_{2}\left\{\int_{0}^{r a_{1} z_{2}} d u \exp \left[-i u-c_{0} r^{-\alpha} u^{\alpha} e^{-i \theta_{0}}\right]\right. \\
& \left(\exp \left[-\tilde{c}_{1}\left(a_{1} z_{2}+\frac{u}{r}\right)^{\alpha} e^{-i \theta_{1}}-\tilde{c}_{2}\left(a_{2} z_{2}+\frac{u}{r}\right)^{\alpha} e^{-i \theta_{2}}-c_{3} z_{2}^{\alpha} e^{-i \theta_{3}}\right]\right. \\
& \left.+\exp \left[-\tilde{c}_{1}\left(a_{1} z_{2}-\frac{u}{r}\right)^{\alpha} e^{i \theta_{1}}-\tilde{c}_{2}\left(a_{2} z_{2}-\frac{u}{r}\right)^{\alpha} e^{i \theta_{2}}-c_{3} z_{2}^{\alpha} e^{i \theta_{3}}\right]\right) \\
& +\int_{r a_{1} z_{2}}^{r a_{2^{z_{2}}}} d u \exp \left[-i u-c_{0} r^{-\alpha} u^{\alpha} e^{-i \theta_{0}}\right] \\
& \left(\exp \left[-\tilde{c}_{1}\left(\frac{u}{r}+a_{1} z_{2}\right)^{\alpha} e^{-i \theta_{1}}-\tilde{c}_{2}\left(a_{2} z_{2}+\frac{u}{r}\right)^{\alpha} e^{-i \theta_{2}}-c_{3} z_{2}^{\alpha} e^{-i \theta_{3}}\right]\right. \\
& \left.+\exp \left[-\tilde{c}_{1}\left(\frac{u}{r}-a_{1} z_{2}\right)^{\alpha} e^{-i \theta_{1}}-\tilde{c}_{2}\left(a_{2} z_{2}-\frac{u}{r}\right)^{\alpha} e^{i \theta_{2}}-c_{3} z_{2}^{\alpha} e^{i \theta_{3}}\right]\right) \\
& +\int_{r a_{2} z_{2}}^{\infty} d u \exp \left[-i u-c_{0} r^{-\alpha} u^{\alpha} e^{-i \theta_{0}}\right]
\end{aligned}
$$




$$
\begin{aligned}
& \left(\exp \left[-\tilde{c}_{1}\left(\frac{u}{r}+a_{1} z_{2}\right)^{\alpha} e^{-i \theta_{1}}-\tilde{c}_{2}\left(\frac{u}{r}+a_{2} z_{2}\right)^{\alpha} e^{-i \theta_{2}}-c_{3} z_{2}^{\alpha} e^{-i \theta}\right]\right. \\
& \left.\left.+\exp \left[-\tilde{c}_{1}\left(\frac{u}{r} a_{1} z_{2}\right)^{\alpha} e^{-i \theta_{1}}-\tilde{c}_{2}\left(\frac{u}{r}-a_{2} z_{2}\right)^{\alpha} e^{-i \theta_{2}}-c_{3} z_{2}^{\alpha} e^{i \theta_{3}}\right]\right)\right\} .
\end{aligned}
$$

First assume $0<\alpha<1$. Rotate the contour of integration with respect to $d u$ through an angle $-\pi / 2$. Then

$$
\begin{aligned}
& 2 \pi^{2} r p(r \sigma)=\operatorname{Re}\left[-i \int_{0}^{\infty} d z_{2}\left\{\int_{0}^{r a_{1} z_{2}} d u e^{-u} \exp \left[-c_{0} r^{-\alpha} u^{\alpha} e^{-i \eta_{0}}\right]\right.\right. \\
& \left(\exp \left[-\tilde{c}_{1}\left(a_{1} z_{2}-i \frac{u}{r}\right)^{\alpha} e^{-i \theta_{1}}-\tilde{c}_{2}\left(a_{2} z_{2}-i \frac{u}{r}\right)^{\alpha} e^{-i \theta_{2}}-c_{3} z_{2}^{\alpha} e^{-i \theta_{3}}\right]\right. \\
& \left.+\exp \left[-\tilde{c}_{1}\left(a_{1} z_{2}+i \frac{u}{r}\right)^{\alpha} e^{i \theta_{1}}-\tilde{c}_{2}\left(a_{2} z_{2}+i \frac{u}{r}\right)^{\alpha} e^{i \theta_{2}}-c_{3} z_{2}^{\alpha} e^{i \theta_{3}}\right]\right) \\
& +\int_{r_{1} a_{2}}^{r a_{2} z_{2}} d u \exp \left[-u-c_{0} r^{-\alpha} u^{\alpha} e^{-i \eta_{0}}\right] \\
& \left(\exp \left[-\tilde{c}_{1}\left(\frac{u}{r}+i a_{1} z_{2}\right)^{\alpha} e^{-i \eta_{1}}-\tilde{c}_{2}\left(a_{2} z_{2}-i \frac{u}{r}\right)^{\alpha} e^{-i \theta_{2}}-c_{3} z_{2}^{\alpha} \epsilon^{-i \theta_{3}}\right]\right. \\
& \left.+\exp \left[-\tilde{c}_{1}\left(\frac{u}{r}-i a_{1} z_{2}\right)^{\alpha} e^{-i \eta_{1}}-\tilde{c}_{2}\left(a_{2} z_{2}+i \frac{u}{r}\right)^{\alpha} e^{i \theta_{2}}-c_{3} z_{2}^{\alpha} e^{i \theta_{3}}\right]\right) \\
& +\int_{r a_{2_{2} z_{2}}}^{\infty} d u \exp \left[-u-c_{0} r^{-\alpha} u^{\alpha} e^{-i \eta_{0}}\right] \\
& \left(\exp \left[-\tilde{c}_{1}\left(\frac{u}{r}+i a_{1} z_{2}\right)^{\alpha} e^{-i \eta_{1}}-\tilde{c}_{2}\left(\frac{u}{r}+i a_{2} z_{2}\right)^{\alpha} e^{-i \eta_{2}}-c_{3} z_{2}^{\alpha} e^{-i \theta_{3}}\right]\right. \\
& \left.\left.+\exp \left[-\tilde{c}_{1}\left(\frac{u}{r}-i a_{1} z_{2}\right)^{\alpha} e^{-i \eta_{1}}-\tilde{c}_{2}\left(\frac{u}{r}-i a_{2} z_{2}\right)^{\alpha} e^{-i \eta_{2}}-c_{3} z_{2}^{\alpha} e^{i \theta_{3}}\right]\right)\right\} \\
& +i \int_{0}^{\infty} d z_{2} r a_{1} z_{2} \int_{0}^{\pi / 2} d \varphi e^{-i \varphi} \\
& \exp \left[-r a_{1} z_{2} e^{i(\pi / 2-\varphi)}-c_{0} a_{1}^{\alpha} z_{2}^{\alpha} e^{-i\left(\theta_{0}+\alpha \varphi\right)}-\tilde{c}_{2} z_{2}^{\alpha}\left(a_{2}-a_{1} e^{-i \varphi}\right)^{\alpha} e^{i \theta_{2}}-c_{3} z_{2}^{\alpha} e^{i \theta_{3}}\right] \\
& \left\{\exp \left[-\tilde{c}_{1} a_{1}^{\alpha} z_{2}^{\alpha}\left(1-e^{-i \varphi}\right)^{\alpha} e^{i \theta_{1}}\right]-\exp \left[-\tilde{c}_{1} a_{1}^{\alpha} z_{2}^{\alpha}\left(e^{-i \varphi}-1\right)^{\alpha} e^{-i \theta_{1}}\right]\right\} \\
& +i \int_{0}^{\infty} d z_{2} r a_{2} z_{2} \int_{0}^{\pi / 2} d \varphi e^{-i \varphi} \\
& \exp \left[-r a_{2} z_{2} e^{i(\pi / 2-\varphi)}-c_{0} a_{2}^{\alpha} z_{2}^{\alpha} e^{-i\left(\theta_{0}+\alpha \varphi\right)}-\tilde{c}_{1} z_{2}^{\alpha}\left(a_{2} e^{-i \varphi}-a_{1}\right)^{\alpha} e^{-i \theta_{1}}-c_{3} z_{2}^{\alpha} e^{i \theta_{3}}\right] \\
& \left.\left\{\exp \left[-\tilde{c}_{2} a_{2}^{\alpha} z_{2}^{\alpha}\left(1-e^{-i \varphi}\right)^{\alpha} e^{i \theta_{2}}\right]-\exp \left[-\tilde{c}_{2} a_{2}^{\alpha} z_{2}^{\alpha}\left(e^{-i \varphi}-1\right)^{\alpha} e^{-i \theta_{2}}\right]\right\}\right] \text {. }
\end{aligned}
$$

In the last two terms change $r a_{1} z_{2}$ and $r a_{2} z_{2}$ to $u, \pi / 2-\varphi$ to $\phi$ respectively and rotate the contour of the integration with respect to $d u$ through an angle $-\phi$. Moreover, in the second and third terms exchange the order of integra- 
tion with respect to $d z_{2}$ and $d u$ and change $r z_{2}$ to $\nu$. Then $2 \pi^{2} r p(r \sigma)$ is equal to

$$
\begin{aligned}
\int_{0}^{\infty} d u \int_{u /\left(r a_{1}\right)}^{\infty} d z e^{-u} \operatorname{Im}\left\{\exp \left[-c_{0} r^{-\alpha} u^{\alpha} e^{-i \eta_{0}}\right]\right\} \\
\quad \operatorname{Re}\left\{\exp \left[-\tilde{c}_{1}\left(a_{1} z_{2}-i \frac{u}{r}\right)^{\alpha} e^{-i \theta_{1}}-\tilde{c}_{2}\left(a_{2} z_{2}-i \frac{u}{r}\right)^{\alpha} e^{-i \theta_{2}}-c_{3} z_{2}^{\alpha} e^{-i \theta_{3}}\right]\right\} \\
\quad+\frac{\operatorname{Im}}{r} \int_{0}^{\infty} d u e^{-u} \exp \left[-c_{0} r^{-\alpha} u^{\alpha} e^{-i \eta_{0}}\right] \\
\quad\left\{\int _ { u / a _ { 2 } } ^ { u / a _ { 1 } } d \nu \left(\exp \left[-\tilde{c}_{1} r^{-\alpha}\left(u+i a_{1} \nu\right)^{\alpha} e^{-i \eta_{1}}-\tilde{c}_{2} r^{-\alpha}\left(a_{2} \nu-i u\right)^{\alpha} e^{-i \theta_{2}}-c_{3} r^{-\alpha} \nu^{\alpha} e^{-i \theta_{3}}\right]\right.\right. \\
\left.+\exp \left[-\tilde{c}_{1} r^{-\alpha}\left(u-i a_{1} \nu\right)^{\alpha} e^{-i \eta_{1}}-\tilde{c}_{2} r^{-\alpha}\left(a_{2} \nu+i u\right)^{\alpha} e^{i \theta_{2}}-c_{3} r^{-\alpha} \nu^{\alpha} e^{i \theta} \varepsilon_{8}\right]\right) \\
\quad+\int_{0}^{u / a_{2}} d \nu\left(\exp \left[-\tilde{c}_{1} r^{-\alpha}\left(u+i a_{1} \nu\right)^{\alpha} e^{-i \eta_{1}}-\tilde{c}_{2} r^{-\alpha}\left(u+i a_{2} \nu\right)^{\alpha} e^{-i \eta_{2}}-c_{3} r^{-\alpha} \nu^{\alpha} e^{-i \theta_{3}}\right]\right. \\
\left.\left.\quad+\exp \left[-\tilde{c}_{1} r^{-\alpha}\left(u-i a_{1} \nu\right)^{\alpha} e^{-i \eta_{1}}-\tilde{c}_{2} r^{-\alpha}\left(u-i a_{2} \nu\right)^{\alpha} e^{-i \eta_{2}}-c_{3} r^{-\alpha} \nu^{\alpha} e^{i \theta_{3}}\right]\right)\right\} \\
\quad+\frac{\operatorname{Re}}{r} \int_{0}^{\infty} d u e^{-u} \exp \left[-c_{0} r^{-\alpha} u^{\alpha} e^{-i \eta_{0}}\right] \frac{u}{a_{1}} \int_{0}^{\pi / 2} d \phi \\
\exp \left[-i \phi-\tilde{c}_{2} r^{-\alpha} u^{\alpha}\left(1-i e^{-i \phi} a_{2} / a_{1}\right)^{\alpha} e^{i \eta_{2}}-c_{3} r^{-\alpha} a_{1}^{-\alpha} u^{\alpha} e^{i\left(\theta_{3}-\alpha \phi\right)}\right] \\
\quad\left\{\exp \left[-\tilde{c}_{1} r^{-\alpha} u^{\alpha}\left(1-i e^{-i \phi}\right)^{\alpha} e^{i \eta_{1}}\right]-\exp \left[-\tilde{c}_{1} r^{-\alpha} u^{\alpha}\left(1-i e^{-i \phi}\right)^{\alpha} e^{-i \eta_{1}}\right]\right\} \\
\quad+\frac{\operatorname{Re}}{r} \int_{0}^{\infty} d u e^{-u} \exp \left[-c_{0} r^{-\alpha} u^{\alpha} e^{-i \eta_{0}}\right] \frac{u}{a_{2}} \int_{0}^{\pi / 2} d \phi \\
\exp \left[-i \phi-\tilde{c}_{1} r^{-\alpha} u^{\alpha}\left(1-i e^{-i \phi} a_{1} / a_{2}\right)^{\alpha} e^{i \eta_{1}}-c_{3} r^{-\alpha} a_{2}^{-\alpha} u^{\alpha} e^{i\left(\theta_{3}-\alpha \phi\right)}\right] \\
\left\{\exp \left[-\tilde{c}_{2} r^{-\alpha} u^{\alpha}\left(1-i e^{-i \phi}\right)^{\alpha} e^{i \eta_{2}}\right]-\exp \left[-\tilde{c}_{2} r^{-\alpha} u^{\alpha}\left(1-i e^{-i \phi}\right)^{\alpha} e^{-i \eta_{2}}\right]\right\} \\
\end{aligned}
$$

Moreover in the second term we see that

$$
\begin{aligned}
& \int_{u / a_{2}}^{u / a_{1}} d \nu\left(\exp \left[\tilde{c}_{1} r^{-\alpha}\left(u+i a_{1} \nu\right)^{\alpha} e^{-i \eta_{1}}-\tilde{c}_{2} r^{-\alpha}\left(a_{2} \nu-i u\right)^{\alpha} e^{-i \theta_{2}}-c_{3} r^{-\alpha} \nu^{\alpha} e^{-i \theta_{3}}\right]\right. \\
& \left.\quad+\exp \left[-\tilde{c}_{1} r^{-\alpha}\left(u-i a_{1} \nu\right)^{\alpha} e^{-i \eta_{1}}-\tilde{c}_{2} r^{-\alpha}\left(a_{2} \nu+i u\right)^{\alpha} e^{i \theta_{2}}-c_{3} r^{-\alpha} \nu^{\alpha} e^{i \theta_{3}}\right]\right) \\
& \quad+\int_{0}^{u / a_{2}} d \nu\left(\exp \left[-\tilde{c}_{1} r^{-\alpha}\left(u+i a_{1} \nu\right)^{\alpha} e^{-i \eta_{1}}-\tilde{c}_{2} r^{-\alpha}\left(u+i a_{2} \nu\right)^{\alpha} e^{-i \eta_{2}}-c_{3} r^{-\alpha} \nu^{\alpha} e^{-i \theta_{3}}\right]\right. \\
& \left.\quad+\exp \left[-\tilde{c}_{1} r^{-\alpha}\left(u-i a_{1} \nu\right)^{\alpha} e^{-i \eta_{1}}-\tilde{c}_{2} r^{-\alpha}\left(u-i a_{2} \nu\right)^{\alpha} e^{-i \eta_{2}}-c_{3} r^{-\alpha} \nu^{\alpha} e^{i \theta_{3}}\right]\right) \\
& =i \int_{u / a_{2}}^{u / a_{1}} d \nu\left(\exp \left[-\tilde{c}_{1} r^{-\alpha}\left(u-a_{1} \nu\right)^{\alpha} e^{-i \eta_{1}}-\tilde{c}_{2} r^{-\alpha}\left(a_{2} \nu-u\right)^{\alpha} e^{i \hat{\eta}_{2}}-c_{3} r^{-\alpha} \nu^{\alpha} e^{i \hat{\eta}_{3}}\right]\right. \\
& \left.\quad-\exp \left[-\tilde{c}_{1} r^{-\alpha}\left(u-a_{1} \nu\right)^{\alpha} e^{-i \eta_{1}}-\tilde{c}_{2} r^{-\alpha}\left(a_{2} \nu-u\right)^{\alpha} e^{-i \hat{\eta}_{2}}-c_{3} r^{-\alpha} \nu^{\alpha} e^{-i \hat{\eta}_{3}}\right]\right) \\
& \quad+i \int_{0}^{u / a_{2}} d \nu\left(\exp \left[-\tilde{c}_{1} r^{-\alpha}\left(u-a_{1} \nu\right)^{\alpha} e^{-i \eta_{1}}-\tilde{c}_{2} r^{-\alpha}\left(u-a_{2} \nu\right)^{\alpha} e^{-i \eta_{2}}-c_{3} r^{-\alpha} \nu^{\alpha} e^{i \hat{\eta}_{3}}\right]\right.
\end{aligned}
$$




$$
\begin{aligned}
& \left.-\exp \left[-\tilde{c}_{1} r^{-\alpha}\left(u-a_{1} \nu\right)^{\alpha} e^{-i \eta_{1}}-\tilde{c}_{2} r^{-\alpha}\left(u-a_{2} \nu\right)^{\alpha} e^{-i \eta_{2}}-c_{3} r^{-\alpha} \nu^{\alpha} e^{-i \hat{\eta}_{3}}\right]\right) \\
& -i \frac{u}{a_{1}} \int_{0}^{\pi / 2} d \phi\left(\operatorname { e x p } \left[i \phi-\tilde{c}_{1} r^{-\alpha} u^{\alpha}\left(1+i e^{i \phi}\right) e^{-i \eta_{1}}\right.\right. \\
& \left.-\tilde{c}_{2} r^{-\alpha} u^{\alpha}\left(1+i e^{i \phi} a_{2} / a_{1}\right)^{\alpha} e^{-i \eta_{2}}-c_{3} r^{-\alpha} a_{1}^{-\alpha} u^{\alpha} e^{-i\left(\theta_{3}-\alpha \phi\right)}\right] \\
& -\exp \left[-i \phi-\tilde{c}_{1} r^{-\alpha} u^{\alpha}\left(1-i e^{-i \phi}\right)^{\alpha} e^{-i \eta_{1}}\right. \\
& \left.\left.-\tilde{c}_{2} r^{-\alpha} u^{\alpha}\left(1-i e^{i \phi} a_{2} / a_{1}\right)^{\alpha} e^{i \eta_{2}}-c_{3} r^{-\alpha} a_{1}^{-\alpha} u^{\alpha} e^{i\left(\theta_{3}-\alpha \phi\right)}\right]\right) \\
& +i \frac{u}{a_{2}} \int_{0}^{\pi / 2} d \phi\left(\operatorname { e x p } \left[i \phi-\tilde{c}_{1} r^{-\alpha} u^{\alpha}\left(1+i e^{i \phi} a_{1} / a_{2}\right)^{\alpha} e^{-i \eta_{1}}\right.\right. \\
& \left.-\tilde{c}_{2} r^{-\alpha} u^{\alpha}\left(1+i e^{i \phi}\right)^{\alpha} e^{-i \eta_{2}}-c_{3} r^{-\alpha} a_{2}^{-\alpha} u^{\alpha} e^{-i\left(\theta_{3}-\alpha \phi\right)}\right] \\
& -\exp \left[-i \phi-\tilde{c}_{1} r^{-\alpha} u^{\alpha}\left(1-i e^{i \phi} a_{1} / a_{2}\right)^{\alpha} e^{-i \eta_{1}}\right. \\
& \left.\left.-\tilde{c}_{2} r^{-\alpha} u^{\alpha}\left(1-i e^{i \phi}\right)^{\alpha} e^{i \eta_{2}}-c_{3} r^{-\alpha} a_{2}^{-\alpha} u^{\alpha} e^{i\left(\theta_{3}-\alpha \phi\right)}\right]\right) \\
& -i \frac{u}{a_{2}} \int_{0}^{\pi / 2} d \phi\left(\operatorname { e x p } \left[i \phi-\tilde{c}_{1} r^{-\alpha} u^{\alpha}\left(1+i e^{i \phi} a_{1} / a_{2}\right)^{\alpha} e^{-i \eta_{1}}\right.\right. \\
& \left.-\tilde{c}_{2} r^{-\alpha} u^{\alpha}\left(1+i e^{i \phi}\right)^{\alpha} e^{-i \eta_{2}}-c_{3} r^{-\alpha} a_{2}^{-\alpha} u^{\alpha} e^{-i\left(\theta_{3}-\alpha \phi\right)}\right] \\
& -\exp \left[-i \phi-\tilde{c}_{1} r^{-\alpha} u^{\alpha}\left(1-i e^{i \phi} a_{1} / a_{2}\right)^{\alpha} e^{-i \eta_{1}}\right. \\
& \left.\left.-\tilde{c}_{2} r^{-\alpha} u^{\alpha}\left(1-i e^{i \phi}\right)^{\alpha} e^{-i \eta_{2}}-c_{3} r^{-\alpha} a_{2}^{-\alpha} u^{\alpha} e^{i\left(\theta_{3}-\alpha \phi\right)}\right]\right)
\end{aligned}
$$

where we rotate the contours through angles $\pm \pi / 2$. Substitute this equation for the above one, then we get

$$
\begin{aligned}
p(r \sigma) & =\frac{1}{\pi^{2} r} \int_{0}^{\infty} d u e^{-u} \operatorname{Im}\left\{\exp \left[-c_{0} r^{-\alpha} u^{\alpha} e^{-i \eta_{0}}\right]\right\} \\
& \operatorname{Re} \int_{u /\left(r a_{1}\right)}^{\infty} d z_{2}\left\{\exp \left[-\tilde{c}_{1}\left(a_{1} z_{2}-i \frac{u}{r}\right)^{\alpha} e^{-i \theta_{1}}-\tilde{c}_{2}\left(a_{2} z_{2}-i \frac{u}{r}\right)^{\alpha} e^{-i \theta_{2}}-c_{3} z_{2}^{\alpha} e^{-i \theta_{3}}\right]\right\} \\
& +\frac{1}{\pi^{2} r^{2}} \int_{0}^{\infty} d u\left\{\int_{u / a_{2}}^{u / a_{1}} d \nu e^{-u} \operatorname{Im}\left\{\exp \left[-c_{0} r^{-\alpha} u^{\alpha} e^{-i \eta_{0}}-\tilde{c}_{1} r^{-\alpha}\left(u-a_{1} \nu\right)^{\alpha} e^{-i \eta_{1}}\right]\right\}\right. \\
& \operatorname{Im}\left\{\exp \left[-\tilde{c}_{2} r^{-\alpha}\left(a_{2} \nu-u\right)^{\alpha} e^{-i \hat{\eta}_{2}}-c_{3} r^{-\alpha} \nu^{\alpha} e^{-i \hat{\eta}_{3}}\right]\right\} \\
& +\int_{0}^{u / a_{2}} d \nu e^{-u} \operatorname{Im}\left\{\operatorname { e x p } \left[-c_{0} r^{-\alpha} u^{\alpha} e^{-i \eta_{0}}-\tilde{c}_{1} r^{-\alpha}\left(u-a_{1} \nu\right)^{\alpha} e^{-i \eta_{1}}\right.\right. \\
& \left.\left.\left.-\tilde{c}_{2} r^{-\alpha}\left(u-a_{2} \nu\right)^{\alpha} e^{-i \eta_{2}}\right]\right\} \operatorname{Im}\left\{\exp \left[-c_{3} r^{-\alpha} \nu^{\alpha} e^{-i \hat{\eta}_{3}}\right]\right\}\right\} \\
& +\frac{1}{\pi^{2} r^{2}} \int_{0}^{\infty} d u e^{-u} \operatorname{Im}\left\{\exp \left[-c_{0} r^{-\alpha} u^{\alpha} e^{-i \eta_{0}}\right]\right\} \frac{u}{a_{1}} \\
& \int_{0}^{\pi / 2} d \phi \exp \left[i \phi-\tilde{c}_{1} r^{-\alpha} u^{\alpha}\left(1+i e^{i \phi}\right)^{\alpha} e^{-i \eta_{1}}\right. \\
& \left.-\tilde{c}_{2} r^{-\alpha} u^{\alpha}\left(1+i e^{i \phi} a_{2} / a_{1}\right)^{\alpha} e^{-i \eta_{2}}-c_{3} r^{-\alpha} a_{1}^{-\alpha} u^{\alpha} e^{-i\left(\theta_{3}-\alpha \phi\right)}\right] .
\end{aligned}
$$


This implies (3.8). Next let $1<\alpha<2$. In this case it is impossible to proceed in the same way as above, because the integral in (3.9) may diverge. However in a similar way to the one-dimensional case (cf. [3] Th. 2.4.2), if we choose suitable angles in the rotation of the contours of integration and use Taylor's formula: For $x>0, y \in \boldsymbol{R}$

$$
\exp [-x+i y]=\sum_{n=0}^{N} \frac{(-x+i y)^{n}}{n !}+\varepsilon \frac{(-x+i y)^{N+1}}{(N+1) !}, \quad \varepsilon \in C,|\varepsilon| \leqq 1,
$$

then we will obtain the same asymptotic expansion (3.8). In fact, first we see that

$$
\begin{aligned}
& 2 \pi^{2} r p(r \sigma)=\operatorname{Re} \int_{0}^{\infty} d z_{2} \int_{0}^{r a_{1} z_{2}} d u \exp \left[-i u-c_{0} r^{-\alpha} u^{\alpha} e^{-\theta_{0}}\right]^{n} / n ! \\
& \left(\exp \left[-\tilde{c}_{1}\left(a_{1} z_{2}+\frac{u}{r}\right)^{\alpha} e^{-i \theta_{1}}-\tilde{c}_{2}\left(a_{2} z_{2}+\frac{u}{r}\right)^{\alpha} e^{-i \theta_{2}}-c_{3} z_{2}^{\alpha} e^{-i \theta_{3}}\right]\right. \\
& \left.+\exp \left[-\tilde{c}_{1}\left(a_{1} z_{2}-\frac{u}{r}\right)^{\alpha} e^{i \theta_{1}}-\tilde{c}_{2}\left(a_{2} z_{2}-\frac{u}{r}\right)^{\alpha} e^{i \theta_{2}}-c_{3} z_{2}^{\alpha} e^{i \theta_{3}}\right]\right) \\
& +\frac{\operatorname{Re}}{r} \int_{0}^{\infty} d \nu\left\{\int_{a_{1} \nu}^{a_{2} \nu} d u \exp \left[-i u-c_{0} r^{-\alpha} u^{\alpha} e^{-i \theta_{0}}\right]\right. \\
& \left(\sum_{n=0}^{N}\left[-\tilde{c}_{1} r^{-\alpha}\left(u+a_{1} \nu\right)^{\alpha} e^{-i \theta_{1}}\right]^{n} / n ! \sum_{n=0}^{N}\left[-\tilde{c}_{2} r^{-\alpha}\left(a_{2} \nu+u\right)^{\alpha} e^{-i \theta_{2}}\right]^{n} / n !\right. \\
& \sum_{n=0}^{N}\left[-c_{3} r^{-\alpha} \nu^{\alpha} e^{-i \theta_{2}}\right]^{n} / n !+\sum_{n=0}^{N}\left[-\tilde{c}_{1} r^{-\alpha}\left(u-a_{1} \nu\right)^{\alpha} e^{-i \theta_{1}}\right]^{n} / n ! \\
& \sum_{n=0}^{N}\left[-\tilde{c}_{2} r^{-\alpha}\left(a_{2} \nu-u\right)^{\alpha} e^{i \theta_{2}}\right]^{n} / n ! \\
& \left.\sum_{n=0}^{N}\left[-c_{3} r^{-\alpha} \nu^{\alpha} e^{i \theta_{3}}\right]^{n} / n !\right)+\int_{a_{2^{\nu}}}^{\infty} d u \exp \left[-i u-c_{0} r^{-\alpha} u^{\alpha} e^{-i \theta_{0}}\right] \\
& \left(\sum_{n=0}^{N}\left[-\tilde{c}_{1} r^{-\alpha}\left(u+a_{1} \nu\right)^{\alpha} e^{-i \theta_{1}}\right]^{n} / n ! \sum_{n=0}^{N}\left[-\tilde{c}_{2} r^{\alpha}\left(u+a_{2} \nu\right)^{\alpha} e^{-i \theta_{2}}\right]^{n} / n !\right. \\
& \sum_{n=0}^{N}\left[-c_{3} r^{-\alpha} \nu^{\alpha} e^{-i \theta_{3}}\right]^{n} / n !+\sum_{n=0}^{N}\left[-\tilde{c}_{1} r^{-\alpha}\left(u-a_{1} \nu\right)^{\alpha} e^{-i \theta_{1}}\right]^{n} / n ! \\
& \left.\left.\sum_{n=0}^{N}\left[-\tilde{c}_{2} r^{-\alpha}\left(u-a_{2} \nu\right)^{\alpha} e^{-i \theta_{2}}\right]^{n} / n ! \sum_{n=0}^{N}\left[-c_{3} r^{-\alpha} \nu^{\alpha} e^{i \theta_{3}}\right]^{n} / n !\right)\right\} \\
& +O\left(r^{-1-(N+1) \alpha}\right) \text {. }
\end{aligned}
$$

In each term we rotate the contour of integration with respect to $d u$ through an angle $\gamma=\pi\left[(\alpha-2) \beta_{0}-1\right] /(2 \alpha)$, then $\exp [-i u]$ is to $\exp \left[-u e^{i(\pi / 2+\gamma)}\right]$ and $\exp \left[-c_{0} r^{-\alpha} u^{\alpha} e^{-i \theta_{0}}\right]$ is to $\exp \left[i c_{0} r^{-\alpha} u^{\alpha}\right]=\sum_{n=0}^{N}\left[i c_{0} r^{-\alpha} u^{\alpha}\right]^{n} / n !+\varepsilon\left[i c_{0} r^{-\alpha} u^{\alpha}\right]^{N+1} /$ $(N+1)$ ! with $\varepsilon \in C,|\varepsilon| \leqq 2$. Note that $-\pi<\gamma<0$ and $|\pi / 2+\gamma|<\pi / 2$. Moreover we rotate the contour through an angle $-\pi / 2-\gamma$. Then we have the expansion which is similar to (3.9). Then by the same way to the case of $0<\alpha<1$ we 
can easily obtain (3.8).

Q.E.D.

Thus if $\sigma \in \mathbf{S p t} \lambda$ and $p_{0}^{\perp}(0)>0$, then

$$
\operatorname{Re} \int_{u / r}^{\infty} \exp \Psi\left(-i \frac{u}{r}, \nu\right) d \nu \longrightarrow \pi p_{0}^{1}(0) \quad \text { as } \quad r \rightarrow+\infty
$$

and

$$
p(r \sigma) \sim r^{-1-\alpha} \pi^{-1} c_{0} \sin \eta_{0} \Gamma(\alpha+1) p_{0}^{1}(0) \quad \text { as } \quad r \rightarrow+\infty .
$$

Therefore we have (a) in Proposition :

If $\sigma \notin$ Spt $\lambda$ then $\beta_{0,0}=-1$, i.e., $\eta_{0}=0$ or $\pi$ (see $\S 2$ ), thus the first and second terms of (3.8) vanish. Hence by change of variables $u-a_{1} \nu$ to $u^{\prime}$ we have the following:

LeMmA 3. Set $b_{j}=a_{j}-a_{1}\left(b_{1}=0, b_{m}=\infty\right)$. Then for $\sigma \notin$ Spt $\lambda$,

$$
\begin{aligned}
& p(r \sigma) \approx r^{-2} \pi^{-2} \sum_{j=1}^{m-1} \int_{0}^{\infty} d \nu \int_{b_{j}^{\nu}}^{b_{j+1} \nu} d u e^{-u-a_{1} \nu} \\
& \begin{array}{c}
\sum_{n=1}^{\infty} \frac{(-1)^{n-1}}{n !} r^{-n \alpha} \operatorname{Im}\left[c_{1} s_{1}^{\alpha} u^{\alpha} e^{-i \eta_{1}}+c_{2} s_{2}^{\alpha}\left(u-b_{2} \nu\right)^{\alpha} e^{-i \eta_{2}}+\cdots\right. \\
\left.\quad+c_{j} s_{j}^{\alpha}\left(u-b_{j} \nu\right)^{\alpha} e^{-i \eta_{j}}\right]^{n}
\end{array} \\
& \sum_{n=1}^{\infty} \frac{(-1)^{n-1}}{n !} r^{-n \alpha} \operatorname{Im}\left[c_{j+1} s_{j+1}^{\alpha}\left(b_{j+1} \nu-u\right)^{\alpha} e^{-i \hat{\eta}_{j+1}}+\cdots+c_{m} \nu^{\alpha} e^{-i \hat{\eta}_{m}}\right]^{n} .
\end{aligned}
$$

as $r \rightarrow+\infty$.

This lemma also holds in the case that $\lambda$ has mass at neither $\sigma$ nor $-\sigma$, because $c_{0}=0$ in (3.8).

Thus if $\sigma \notin$ Spt $\lambda$ and $\sigma \in \operatorname{Int} S(2)$, then

$$
\begin{aligned}
p(r \sigma) & \sim r^{-2(1+\alpha)} \pi^{-2} \Gamma(\alpha+1)^{2} \sum_{1 \leq j<k \leq m} g_{j, k} c_{j}\left|h_{j, k}\right|^{-1-\alpha} \sin \eta_{j} c_{k}\left|h_{k, j}\right|^{-1-\alpha} \sin \hat{\eta}_{k} \\
& \sim \sum_{1 \leq j<k \leq m} g_{j, k} p_{j}\left(r h_{j, k}\right) p_{k}\left(r h_{k, j}\right) \quad \text { as } r \rightarrow+\infty \\
& =\sum_{1 \leq j<k \leq m} p_{j, k}(r \sigma),
\end{aligned}
$$

where $g_{j, k}=\left(s_{j} t_{k}-s_{k} t_{j}\right)^{-1}>0$ for $j<k, h_{j, k}$ and $h_{k, j}$ are defined by $\sigma=h_{j, k} \sigma_{j}+$ $h_{k, j} \sigma_{k}$ (i.e., $\left.h_{j, k}=t_{k} /\left(s_{j} t_{k}-s_{k} t_{j}\right)\right)$. Thus, we get (b) in Proposition.

Moreover if $1<\alpha<2$ and $\sigma \notin S(2)$, then $\beta_{1,0}=\cdots=\beta_{m, 0}= \pm 1$ (i.e., $\hat{\eta}_{1}=\cdots=\hat{\eta}_{m}$ $=\pi$ or $\left.\eta_{1}=\cdots=\eta_{m}=\pi\right)$. Hence every term of (3.10) vanish. We have (c) in Proposition.

Finally (d) is followed by Lemma 2 .

Second step. Suppose that $\lambda$ has mass at only $(m+1)$-directions $\sigma_{j}, j=0,1$, $2, \cdots, m$. We may assume that $\sigma=(1,0)$ and $0 \leqq \varphi_{0}<\varphi_{1}<\varphi_{2}<\cdots<\varphi_{m-1}<\varphi_{m}<$ 
$\pi\left(\varphi_{j}=\arg \sigma_{j}\right)$. If $\lambda$ has no mass at $\{ \pm \sigma\}$, then by taking $c_{0}=0$ in $\Psi(z)$ and seting $\sigma_{0}=\sigma$ we may include $\sigma$ as a member of directions $\sigma_{j}, j=0,1, \cdots, m$. Moreover in (3.1) let $Q$ be a linear transformation such that $Q \sigma_{0}=\sigma_{0}$ and $Q \sigma_{m}$ $=(0,1)$, then $0=\tilde{\varphi}_{0}<\tilde{\varphi}_{1}<\cdots<\tilde{\varphi}_{m}=\pi / 2$ where $\tilde{\varphi}_{j}=\arg Q \sigma_{j}$. Thus by $Q r \sigma=r \sigma$ we have $p_{\lambda}(r \sigma)=|\operatorname{det} Q| p_{\lambda_{Q}}(r \sigma)$ and $\lambda_{Q}$ has mass at only $(m+1)$-directions $Q \sigma_{j}$, $j=0,1, \cdots, m$. Therefore the general case is reduced to the special case of First step.

The proof of Theorem 2 and Theorem 3 in Case 2 is complete.

CASE 3. $\alpha=1$ and $\lambda$ has mass at $(m+1)$-directions $\sigma_{0}, \sigma_{1}, \cdots, \sigma_{m}(m \geqq 2)$. We may also take $\left\{\sigma_{j}, j=0,1,2, \cdots, m\right\}$ as in First step of Case 2. Then for $z=\left(z_{1}, z_{2}\right) \in \boldsymbol{R}^{2}$

$$
\Psi(z)=-\sum_{j=0}^{m} c_{j}\left\{\left|\left\langle\sigma_{j}, z\right\rangle\right|+i \frac{2}{\pi} \beta_{j}\left\langle\sigma_{j}, z\right\rangle \log \left|\left\langle\sigma_{j}, z\right\rangle\right|\right\},
$$

where $c_{j}>0,\left|\beta_{j}\right| \leqq 1, j=0,1,2, \cdots, m$ are constants.

The following lemma is corresponding to Lemma 2 and Lemma 3.

LEMMA 4. Let $r \geqq 0$ and $\sigma=\sigma_{0}=(1,0)$.

(i) Then for $a_{j}=\tan \phi_{j}$

$$
\begin{aligned}
p(r \boldsymbol{\sigma}) & =(2 \pi)^{-2} \int_{R^{2}} \exp \left[-i r z_{1}+\Psi(z)\right] d z \\
& \approx r^{-1} \pi^{-2} \sum_{n=1}^{\infty} \frac{r^{-n}}{n !} c_{0}^{n} \int_{0}^{\infty} e^{-u} u^{n} \operatorname{Im}\left[i\left(1+\beta_{0}\right)-\frac{2 \beta_{0}}{\pi} \log \frac{u}{r}\right]^{n} d u \\
& \operatorname{Re} \int_{u / r}^{\infty} \exp \Psi\left(-i \frac{u}{r}, \nu\right) d \nu \\
& +r^{2} \pi^{-2} \sum_{n=1}^{\infty} \frac{r^{-n}}{n !} c_{0}^{n} \int_{0}^{\infty} e^{-u} u^{n+1} \operatorname{Im}\left[i\left(1+\beta_{0}\right)-\frac{2}{\pi} \beta_{0} \log \frac{u}{r}\right]^{n} d u \\
& +r^{-2} \pi^{-2} \sum_{j=1}^{m-1} \int_{0}^{\infty} d \nu \int_{a_{j} \nu}^{a_{j+1} \nu} d u e^{-u} \sum_{n=1}^{\infty} \frac{r^{-n}}{n !} \operatorname{Im}\left[c_{0} u\left\{i\left(1+\beta_{0}\right)-\frac{2}{\pi} \beta_{0} \log \frac{u}{r}\right\}\right. \\
& +c_{1} s_{1}\left(u-a_{1} \nu\right)\left\{i\left(1+\beta_{1}\right)-\frac{2}{\pi} \beta_{1} \log \left[s_{1}\left(u-a_{1} \nu\right) / r\right]\right\}+\cdots \\
& +c_{j} s_{j}\left(u-a_{j \nu} \nu\left\{i\left(1+\beta_{j}\right)-\frac{2}{\pi} \beta_{j} \log \left[s_{j}\left(u-a_{j} \nu\right) / r\right]\right\}\right]^{n} \\
& \sum_{n=1}^{\infty} \frac{r^{-n}}{n !} \operatorname{Im}\left[c_{j+1} s_{j+1}\left(a_{j+1} \nu-u\right)\left\{i\left(1-\beta_{j+1}\right)+\frac{2}{\pi} \beta_{j+1} \log \left[s_{j+1}\left(a_{j+1} \nu-u\right) / r\right]\right\}\right.
\end{aligned}
$$




$$
\left.+\cdots+c_{m} \nu\left\{i\left(1-\beta_{m}\right)+\frac{2}{\pi} \beta_{m} \log \frac{\nu}{r}\right\}\right]^{n}
$$

as $r \rightarrow+\infty$.

(ii) If $\sigma \notin$ Spt $\lambda$, set $b_{5}=a_{5}-a_{1}$, then

$$
\begin{gathered}
p(r \sigma) \approx r^{-2} \pi^{-2} \sum_{j=1}^{m-1} \int_{0}^{\infty} d \nu \int_{b_{j} \nu}^{b_{j+1} \nu} d u e^{-u-a_{1} \nu} \\
\sum_{n=1}^{\infty} \frac{r^{-n}}{n !} \operatorname{Im}\left[c_{1} s_{1} u\left\{i\left(1+\beta_{1}\right)-\frac{2}{\pi} \beta_{1} \log \left[s_{1}\left(u-b_{1}\right) / r\right]\right\}+\cdots\right. \\
\left.\quad+c_{j} s_{j}\left(u-b_{j} \nu\right)\left\{i\left(1+\beta_{j}\right)-\frac{2}{\pi} \beta_{j} \log \left[s_{j}\left(u-b_{j} \nu\right) / r\right]\right\}\right]^{n} \\
\sum_{n=1}^{\infty} \frac{r^{-n}}{n !} \operatorname{Im}\left[c_{j+1} s_{j+1}\left(b_{j+1} \nu-u\right)\left\{i\left(1-\beta_{j+1}\right)+\frac{2}{\pi} \beta_{j+1} \log \left[s_{j+1}\left(b_{j+1} \nu-u\right) / r\right]\right\}\right. \\
\left.+\cdots+c_{m} \nu\left\{i\left(1-\beta_{m}\right)+\frac{2}{\pi} \beta_{m} \log \frac{\nu}{r}\right\}\right]^{n}
\end{gathered}
$$

as $r \rightarrow+\infty$.

From this lemma we obtain Theorem 2 and Theorem 3 by the same way as in case of $1<\alpha<2$.

Next we proceed the proof of Theorem 2 in case of $d=3$.

(1) First we see that

$$
\begin{aligned}
(2 \pi)^{3} p(x)=\int_{R^{3}} \exp [-i\langle x, z\rangle+\Psi(z)] d z \\
=2 \operatorname{Re} \int_{R_{+}^{3}} d z\left\{\exp \left[-i\left(x_{1} z_{1}+x_{2} z_{2}+x_{3} z_{3}\right)+\Psi\left(z_{1}, z_{2}, z_{3}\right)\right]\right. \\
\quad+\exp \left[-i\left(x_{1} z_{1}-x_{2} z_{2}+x_{3} z_{3}\right)+\Psi\left(z^{\mathrm{I}},-z_{2}, z_{3}\right)\right] \\
\quad+\exp \left[-i\left(x_{1} z_{1}+x_{2} z_{2}-x_{3} z_{3}\right)+\Psi\left(z_{1}, z_{2},-z_{3}\right)\right] \\
\left.\quad+\exp \left[-i\left(x_{1} z_{1}-x_{2} z_{2}-x_{3} z_{3}\right)+\Psi\left(z_{1},-z_{2},-z_{3}\right)\right]\right\} .
\end{aligned}
$$

(2) We divide the integral domain in order to omit the notation "sgn" in $\Psi(z)$.

(3) We change variables $z_{1}, z_{2}, z_{3}$ appropriately according to $\sigma$.

Then we deduce that Theorem 2 and Theorem 3 hold. We will describe the outline of the proof in some details. Here we only consider the case that $\lambda$ has mass at $(m+1)$-directions $\sigma_{0}, \sigma_{1}, \cdots \sigma_{m}(m \geqq 3)$ but that $0<\alpha<1$ and $\sigma \notin$ Int $S(3)$, because it is evident in the others.

a) If $\sigma \in T(1)$, i.e., $\lambda(\{\sigma\})>0$, we may take $\sigma=\sigma_{0}=(1,0,0)$ and change $z_{1}$ to $-i u / r$, then we have $p(r \sigma) \sim p_{0}(r) p_{0}^{1}(0) \sim c r^{-1-\alpha}(c>0)$ as $r \rightarrow+\infty$. 
EXAMPLE 1. Let $m=5, \sigma=\sigma_{0}=(1,0,0), \sigma_{1}=(0,1,0), \sigma_{2}=(1 / \sqrt{3}, 1 / \sqrt{3}$, $1 / \sqrt{3}), \sigma_{3}=(0,1 / \sqrt{2}, 1 / \sqrt{2}), \sigma_{4}=(1 / \sqrt{2}, 0,1 / \sqrt{2})$ and $\sigma_{5}=(0,0,1)$. In (3.11) we divide the integral domain as follows:

$$
\begin{aligned}
\int_{R_{+}^{3}} d z=\int_{0}^{\infty} d z_{3} & \left\{\int_{0}^{z_{3} / 2} d z_{2}\left(\int_{0}^{z_{2}} d z_{2} \int_{z_{2}}^{z_{3}-z_{2}}+\int_{z_{3}-z_{2}}^{z_{3}}+\int_{z_{3}}^{z_{3}+z_{2}}+\int_{z_{3}+z_{2}}^{\infty} d z_{1}\right)\right. \\
& +\int_{z_{3} / 2}^{z_{3}} d z_{2}\left(\int_{0}^{z_{3}-z_{2}}+\int_{z_{3}-z_{2}}^{z_{2}}+\int_{z_{2}}^{z_{3}}+\int_{z_{2}}^{z_{3}+z_{2}}+\int_{z_{3}+z_{2}}^{\infty} d z_{1}\right) \\
& +\int_{z_{3}}^{2 z_{3}} d z_{2}\left(\int_{0}^{z_{2}-z_{3}}+\int_{z_{2}-z_{3}}^{z_{3}}+\int_{z_{3}}^{z_{2}}+\int_{z_{2}}^{z_{2}+z_{3}}+\int_{z_{2}+z_{3}}^{\infty} d z_{1}\right) \\
& \left.+\int_{2 z_{3}}^{\infty} d z_{2}\left(\int_{0}^{z_{3}}+\int_{z_{3}}^{z_{2}-z_{3}}+\int_{z_{2}-z_{3}}^{z_{2}}+\int_{z_{2}}^{z_{2}+z_{3}}+\int_{z_{2}+z_{3}}^{\infty} d z_{1}\right)\right\}
\end{aligned}
$$

and change $z_{1}$ to $-i u / r$, then we can see that the sum of terms in (3.11) corresponding to the first integral with respect to $d z_{1}$ of each term in (3.12) decreases like $p_{0}(r) p_{0}^{\perp}(0) \sim c r^{-1-\alpha}(c>0)$ as $r \rightarrow+\infty$. Moreover, the remaining terms are $o\left(r^{-1-\alpha}\right)$ as $r \rightarrow+\infty$.

b) If $\sigma \in T(2)$, then the following two cases arise.

(i) There exists only one plane $H$ which is spanned by some elements $\sigma_{0}, \sigma_{1}, \cdots, \sigma_{k}(k \geqq 1)$ of Spt $\lambda$ and contains $\sigma$. In this case we may assume that $H$ is $x_{1} x_{2}$-plane, $\sigma=(1 / \sqrt{2}, 1 / \sqrt{2}, 0), \sigma_{0}=(1,0,0), \sigma_{1}=(0,1,0)$ and $\sigma_{2}, \cdots$, $\sigma_{k} \in\left\{\theta_{3}=0\right\} \backslash\left\{\theta_{1} \geqq 0, \theta_{2} \geqq 0, \theta_{3}=0\right\}$ in $S^{2}$. Set $r^{\prime}=r / \sqrt{2}$. We divide the integral domain as mentioned in (2) and change $\left(z_{1}, \pm z_{2}\right)$ to $-i\left(u_{1} / r^{\prime}, \pm u_{2} / r^{\prime}\right)$ in order to $\exp \left[-i r^{\prime}\left(z_{1} \pm z_{2}\right)\right]$ become $\exp \left[-u_{1}-u_{2}\right]$ in (3.11). Then we have an asymptotic behaviour $p(r \sigma) \sim r^{-2(1+\alpha)}$ as $r \rightarrow+\infty$.

EXAMPLE 2. Let $m=3, k=1, \sigma_{0}=(1,0,0), \sigma_{1}=(0,1,0), \sigma_{2}=(0,1 / \sqrt{5}$, $2 / \sqrt{5}), \sigma_{3}=(0,0,1)$ and $\sigma=(1 / \sqrt{2}, 1 / \sqrt{2}, 0) \in \operatorname{Con}\left\{\sigma_{0}, \sigma_{1}\right\}$. In (3.11) we divide the integral as follows:

$$
\int_{R_{+}^{3}} d z=\int_{0}^{\infty} d z_{3} \int_{0}^{\infty} d z_{1}\left\{\int_{0}^{2 z_{3}}+\int_{2 z_{3}}^{\infty} d z_{2}\right\} .
$$

Change variables $z_{1}$ and $z_{2}$. Then from the term in (3.11) corresponding to the first integral in the above we have an asymptotic $p_{0,1}(r \sigma(0,1)) p_{0,1}^{1}(0)\left(\sim c r^{-2(1+\alpha)}\right.$, $c>0)$ as $r \rightarrow+\infty$, where $\sigma(0,1)$ is a restriction of $\sigma$ to $\operatorname{Span}\left\{\sigma_{0}, \sigma_{1}\right\}$. Moreover, from the other we have $o\left(r^{-2(1+\alpha)}\right)$ as $r \rightarrow+\infty$.

(ii) There exist at least two planes $H_{1}, H_{2}$ which are spanned by some elements of Spt $\lambda$ and $H_{1} \cap H_{2}$ is a line containing $\sigma$. In this case we take $\sigma$ $=(1,0,0)$. We change $z_{1}$ to $-i u_{1} / r$ and also $z_{2}$ appropriately as seen in the following example. Then we have $p(r \sigma) \sim r^{-2(1+\alpha)}$ as $r \rightarrow+\infty$. 
EXAMPLE 3. The setting is the same as in Example 1 except $\sigma_{0}=(1 / \sqrt{2}$, $1 / \sqrt{2}, 0) \neq \sigma=(1,0,0)$, and also divide the integral domain as in it. First in each integral we change $z_{1}$ to $-i u_{1} / r$ then in (3.11) terms vanish which correspond to the first integrals with respect to $z_{1}$ in (3.12). In the integral $\int_{0}^{\infty} d z_{3} \int_{0}^{z_{3} / 2} d z_{2} \int_{r z_{2}}^{r\left(z_{3}-z_{2}\right)} d u_{1}$ we change $z_{2}$ to $+i u_{2} / r,-i u_{2} / r,+i u_{2} / r$ and $-i u_{2} / r$ according to each term of (3.11). Then we have the asymptotic $p_{0,1}(r \sigma(0,1))$ $p_{0,1}^{1}(0)$ as $r \rightarrow+\infty$. Moreover by the same change of variables we have $o\left(r^{-2(1+\alpha)}\right)$ as $r \rightarrow+\infty$ from the integrals of

$$
\begin{array}{r}
\int_{0}^{\infty} d z_{3}\left\{\int_{0}^{z_{3} / 2} d z_{2}\left(\int_{r\left(z_{3}+z_{2}\right)}^{r z_{3}}+\int_{r z_{3}}^{r\left(z_{3}+z_{2}\right)}+\int_{r\left(z_{3}+z_{2}\right)}^{\infty} d u_{1}\right)\right. \\
\left.+\int_{z_{3} / 2}^{z_{3}} d z_{2}\left(\int_{r z_{2}}^{r z_{3}}+\int_{r z_{3}}^{r\left(z_{3}+z_{2}\right)}+\int_{r\left(z_{2}+z_{3}\right)}^{\infty} d u_{1}\right)\right\} .
\end{array}
$$

Similarly, in the integral $\int_{0}^{\infty} d z_{3} \int_{2 z_{3}}^{\infty} d z_{2} \int_{r z_{3}}^{r\left(z_{2}-z_{3}\right)} d u_{1}$ we change $z_{3}$ to $+i u_{3} / r,+i u_{3} / r$, $-i u_{3} / r$ and $-i u_{3} / r$ according to each term of (3.11). Then we have the asymptotic $p_{4,5}(r \sigma(4,5)) p_{4,5}^{1}(0)$ as $r \rightarrow+\infty$, and by the same change of variables we have $o\left(r^{-2(1+\alpha)}\right)$ as $r \rightarrow+\infty$ from the integrals of

$$
\begin{aligned}
& \int_{0}^{\infty} d z_{3}\left\{\int_{z_{3}}^{z_{3}} d z_{2}\left(\int_{r z_{3}}^{r z_{2}}+\int_{r z_{2}}^{r\left(z_{2}+z_{3}\right)}+\int_{r\left(z_{2}+z_{3}\right)}^{\infty} d u_{1}\right)\right. \\
& \left.\quad+\int_{z z_{3}}^{\infty} d z_{2}\left(\int_{r\left(z_{2}-z_{3}\right)}^{r z_{2}}+\int_{r z_{2}}^{r\left(z_{2}+z_{3}\right)}+\int_{r\left(z_{2}+z_{3}\right)}^{\infty} d u_{1}\right)\right\} \\
& =\int_{0}^{\infty} d z_{2}\left\{\int_{z_{2} / 2}^{z_{2}} d z_{2}\left(\int_{r z_{3}}^{r z_{2}}+\int_{r z_{2}}^{r\left(z_{2}+z_{3}\right)}+\int_{r\left(z_{2}+z_{3}\right)}^{\infty} d u_{1}\right)\right. \\
& \left.+\int_{0}^{z_{2} / 2} d z_{3}\left(\int_{r\left(z_{2}-z_{3}\right)}^{r z_{2}}+\int_{r z_{2}}^{r\left(z_{2}+z_{3}\right)}+\int_{r\left(z_{2}+z_{3}\right)}^{\infty} d u_{1}\right)\right\} .
\end{aligned}
$$

Finally we have the asymptotic $p_{2,3}(r \sigma(2,3)) p_{2,3}^{1}(0)$ as $r \rightarrow+\infty$ from the remaining terms. In fact, in the integral $\int_{0}^{\infty} d z_{3} \int_{z_{3} / 2}^{z_{3}} d z_{2} \int_{r_{3}}^{r_{2}} d u_{1}$ we change $z_{2}$ (resp. $z_{3}$ ) to $-i u_{2} / r,+i u_{2} / r,-i u_{2} / r$ and $+i u_{2} / r\left(\right.$ resp. $+i u_{3} / r,+i u_{3} / r,-i u_{3} / r$ and $\left.-i u_{3} / r\right)$ according to each term of (3.11). Moreover change variables $\left(u_{1}, u_{2}, u_{3}\right)$ to $\left(\nu_{1}+\nu_{3}, \nu_{2}, \nu_{2}+\nu_{3}\right)$. Then the sum of the first and 4 -th terms vanish and we change $\nu_{2}$ to $-i \nu_{2}$ (resp. $+i \nu_{2}$ ) in the second term (resp. third term). Similarly in $\int_{0}^{\infty} d z_{3} \int_{z_{2}}^{2 z_{3}} d z_{2} \int_{r\left(z_{2}-z_{3}\right)}^{r z_{3}} d u_{1}$ change $z_{2}$ (resp. $z_{3}$ ) to $+i u_{2} / r,-i u_{2} / r,+i u_{2} / r$ and $-i u_{2} / r$ (resp. $-i u_{3} / r,-i u_{3} / r,+i u_{3} / r$ and $+i u_{3} / r$ ) according to each terms of (3.11), and $\left(u_{1}, u_{2}, u_{3}\right)$ to $\left(\nu_{1}+\nu_{2}, \nu_{2}+\nu_{3}, \nu_{3}\right)$. Then the sum of the first and 4 -th terms vanish. Hence, we change $\nu_{3}$ to $+i \nu_{3}$ (resp. $-i \nu_{3}$ ) in the second term 
(resp. third term). By this way we have $p_{2,3}(r \sigma(2,3)) p_{2,3}^{\frac{1}{3}}(0)$ as $r \rightarrow+\infty$. Therefore we see that $p(r \sigma) \sim p_{0,1}(r \sigma(0,1)) p_{0,1}^{\perp}(0)+p_{2,3}(r \sigma(2,3)) p_{2,3}^{\frac{1}{3}}(0)+p_{4,5}(r \sigma(4,5))$ $p_{4,5}^{1}(0)\left(\sim c r^{-2(1+\alpha)}\right)$ as $r \rightarrow+\infty$.

c) If $\sigma \in T(3)$, it is sufficient to consider the case that $\sigma=(1 / \sqrt{3}, 1 / \sqrt{3}$, $1 / \sqrt{3}), \sigma_{0}=(1,0,0), \sigma_{1}=(0,1,0), \sigma_{2}=(0,0,1)$ and $\sigma_{3}, \cdots, \sigma_{m} \subset S^{2} \backslash\left\{\theta_{1} \geqq 0, \theta_{2} \geqq 0\right.$, $\left.\theta_{3} \geqq 0\right\}$. Set $r^{\prime}=r / \sqrt{3}$. We divide the integral domain as mentioned in (2) and change $\left(z_{1}, z_{2}, z_{3}\right)$ to $-i\left(u_{1} / r^{\prime}, \pm u_{2} / r^{\prime}, \pm u_{3} / r^{\prime}\right)$ in order to $\exp \left[-i r^{\prime}\left(z_{1} \pm z_{2} \pm z_{3}\right)\right]$ be to $\exp \left[-u_{1}-u_{2}-u_{3}\right]$ in (3.11). For instance, for $\exp \left[-i r^{\prime}\left(z_{1}-z_{2}+z_{3}\right]\right)$ we change $\left(z_{1}, z_{2}, z_{3}\right)$ to $-i\left(u_{1} / r^{\prime},-u_{2} / r^{\prime}, u_{3} / r^{\prime}\right)$. Then we have an asymptoic $p(r \sigma)$ $\sim r^{-3(1+\alpha)}$ as $r \rightarrow+\infty$.

EXAMPLE 4. Let $m=3, \sigma_{0}=(1,0,0), \sigma_{1}=(0,1,0), \sigma_{2}=(0,0,1), \sigma_{3}=(0$, $-1 / \sqrt{2}, 1 / \sqrt{2})$ and $\sigma=(1 / \sqrt{3}, 1 / \sqrt{3}, 1 / \sqrt{3})$. In (3.11) we divide the integral

$$
\int_{R_{+}^{3}} d z=\int_{0}^{\infty} d z_{3} \int_{0}^{\infty} d z_{1}\left\{\int_{0}^{z_{3}}+\int_{z_{3}}^{\infty} d z_{2}\right\}
$$

Change variables $z_{1}, z_{2}$ and $z_{3}$ as above. Then we can easily deduce that $p(r \sigma) \sim p_{0,1,2}(r \sigma) p_{0,1,2}^{\perp}(0)+p_{0,1,3}(r \sigma) p_{0,1,3}^{\perp}(0)+p_{0,2,3}(r \sigma) p_{0,2,3}^{\perp}(0)\left(\sim c r^{-3(1+\alpha)}, c>0\right)$ as $r \rightarrow+\infty$.

d) If $\sigma \in S(3)$ and $1 \leqq \alpha<2$, then by the same way as in (c) we can see that $p(r \sigma)$ is rapidly decreasing as $r \rightarrow+\infty$.

All of the above change of variables are informal, however we can justify the computations by a similar way to the case of $d=2$.

Then we conclude Theorem 2 and Theorem 3.

REMARK 4. As mentioned in $\S 1$, in higher dimensions $(d \geqq 4)$ we belive that our method should work, although the calculations may be more tedious and complicated.

\section{Acknowledgements}

The author would like to thank Professor T. Shiga for his valuable suggestions and teachings. He also wishes to thank the refree for his useful suggestions and comments.

\section{References}

[1] Arkhipov, S.V., The density function's asymptotic representation in the case of multidimensional strictly stable distributions, Lecture Notes in Math., 1412 (1987), 1-21, Springer-Verlag, Berlin. 
[2] Dziubanski, J., Asymptotic behaviour of densities of stable semigroups of measures, Probab. Th. Rel. Fields, 87 (1990), 459-467.

[3] Ibragimov, 1. A. and Linnik, Yu., Independent and stationary sequences of random variables, Wolters-Noordhoff, Groningen, 1971.

[4] Ikeda, N. and Watanabe, S., Stochastic differential equations and diffusion processes, New York Amsterdam, North-Holland/Kodansha, 1981.

[5] Port, S.C., Asymptotic expansions for the expected volume of a stable sausage, Ann. Probab., 18-2 (1990), 492-523.

[6] Pruitt, W.E. and Taylor, S.J., The potential kernel and hitting probabilities for the general stable process in $R^{n}$, Trans. Amer. Math. Soc., 146 (1969), 299321.

[7] Sato, K., Class $L$ of multivariate distributions and subclasses, J. Multivar. Anal., 10-2 (1980), 207-232.

[8] Sato, K., Processes with independent increments, (in Japanese) Kinokuniya Company Limited, 1990.

[9] Taylor, S. J., Sample path properties of a transient stable process, J. Mech., 16 (1967), 1229-1246.

Department of Mathematics

Osaka City University

Sugimoto-3, Sumiyoshi-ku

Osaka 558, Japan

E-mail address:

h1753(C)c. osaka-cu. ac. jp 